\title{
Economic and Humanistic Burden of Triple-Negative Breast Cancer: A Systematic Literature Review
}

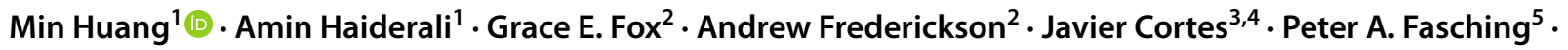 \\ Joyce O'Shaughnessy ${ }^{6}$
}

Accepted: 28 November 2021 / Published online: 3 February 2022

(c) The Author(s) 2022

\begin{abstract}
Background Triple-negative breast cancer (TNBC) accounts for 10-20\% of all breast cancers (BCs). It is more commonly diagnosed in younger women and often has a less favorable prognosis compared with other BC subtypes.

Objective The objective of this study was to provide a literature-based extensive overview of the economic and humanistic burden of TNBC to assist medical decisions for healthcare payers, providers, and patients.

Methods A systematic literature review was performed using multiple databases, including EMBASE, MEDLINE, Econlit, the Cochrane Central Register of Controlled Trials, and the Cochrane Database of Systematic Reviews, from database inception to 16 May 2021. In addition, a targeted search was performed in the Northern Light Life Sciences Conference Abstracts database from 2016 through June 2021. The bibliographies of included articles were reviewed to identify other potentially relevant publications. Quality assessment of the included studies was conducted.

Results The review identified 19 studies assessing the economic burden and 10 studies assessing the humanistic burden of TNBC. Studies varied widely in study design, settings, patient populations, and time horizons. The estimates of mean perpatient annual direct medical costs ranged from around $\$ 20,000$ to over $\$ 100,000$ in stage I-III TNBC and from $\$ 100,000$ to $\$ 300,000$ in stage IV TNBC. Healthcare costs and resource utilization increased significantly with disease recurrence, progression, and increased cancer stage or line of therapy. Compared with the costs of systemic anticancer therapy, cancer management costs comprised a larger portion of total direct costs. The estimates of indirect costs due to productivity loss ranged from $\$ 207$ to $\$ 1573$ per patient per month (all costs presented above were adjusted to 2021 US dollars). Cancer recurrence led to significantly reduced productivity and greater rates of leaving the workforce. A rapid deterioration of health utility associated with disease progression was observed in TNBC patients. Treatment with pembrolizumab or talazoparib showed significantly greater improvements in health-related quality of life (HRQoL) compared with chemotherapy, as measured by EORTC QLQ-C30, QLQ-BR23, and FACT-B.

Conclusion TNBC is associated with a substantial economic burden on healthcare systems and societies and considerably reduced productivity and HRQoL for patients. This study synthesized the published literature on the economic and humanistic burden of TNBC and highlighted the need for continued research due to the rapidly changing landscape of TNBC care.
\end{abstract}

\section{Introduction}

With an estimated 2.26 million new cases in 2020, breast cancer (BC) is the most common cancer diagnosed globally. It is the fifth leading cause of cancer deaths worldwide and is estimated to have caused 684,996 deaths in 2020 [1]. Triplenegative breast cancer (TNBC) [2], a molecular phenotype of $\mathrm{BC}$ characterized by the absence of estrogen receptor

Min Huang

Min_Huang@merck.com

Extended author information available on the last page of the article
(ER), progesterone receptor (PR), and human epidermal growth factor receptor-2 (HER2) expression, accounts for nearly $10-20 \%$ of all BCs [3]. TNBC is more commonly diagnosed in younger women and has a higher risk of distant relapse in the first few years after diagnosis than other types of BC [4].

Past treatment options for TNBC patients were limited and the established systemic treatment was mainly chemotherapy. The treatment landscape has expanded with the recent regulatory approval of immuno-oncology agents in both early stage and metastatic settings [5-7]. The National Comprehensive Care Network (NCCN) guideline recommends pembrolizumab in combination with chemotherapy 


\section{Key Points for Decision Makers}

Triple-negative breast cancer (TNBC) is associated with a significant economic burden, with substantially greater costs associated with increasing disease severity. TNBC patients experience decreased work productivity, reduced HRQoL, and rising out-of-pocket expenses.

Many studies reported that emergency department visits and hospitalizations were the main cost drivers in metastatic TNBC, while anticancer systemic therapies accounted for only a small portion of the total medical costs. This finding suggests that focusing on hospital and terminal care is integral to managing overall costs in the late stage of the disease.

With the emergence of new cancer therapies, especially immuno-oncology treatments for TNBC, additional research is required to evaluate the impact of these therapies on the economic and humanistic burden of the disease to assist medical decisions for healthcare payers, providers, and patients.

as neoadjuvant treatment followed by pembrolizumab as a single agent as adjuvant treatment for patients with highrisk, early-stage TNBC (eTNBC). Chemotherapies with a mix of regimens comprising chemotherapeutic drugs from several classes, including anthracyclines, alkylating agents, and taxanes are recommended by the NCCN and the European Society for Medical Oncology (ESMO) guidelines as neoadjuvant or adjuvant treatment for patients with eTNBC [8-10]. In patients with locally recurrent unresectable or metastatic TNBC (mTNBC) with programmed cell death ligand 1 (PD-L1)-positive tumors, the guidelines recommend pembrolizumab in combination with chemotherapy (including paclitaxel, nab-paclitaxel, or gemcitabine plus carboplatin). Atezolizumab in combination with nab-paclitaxel is recommended by the ESMO guideline as first-line treatment in this population. Platinum-based chemotherapy and the targeted therapies olaparib and talazoparib are recommended for patients with BRCA mutations. Furthermore, the anti-Trop2 antibody-drug conjugate sacituzumab govitecan-hziy is recommended for patients who have received at least two prior lines of treatment for mTNBC.

Research over the last few decades has underscored the substantial economic burden of BC, especially advanced or metastatic BC [11-13, 15]. The overall economic impact of $\mathrm{BC}$ was estimated to be approximately $\$ 88$ billion globally in the year 2010 [14]. Patients with TNBC are generally diagnosed at later stages, have a poorer prognosis, a higher risk of recurrence, and incur higher hospital resource use and higher costs of care compared with those of nonTNBC subtypes [16]. Due to the increasing incidence and prolonged patient survival, combined with higher unit costs of cancer care, the economic burden of BC has most likely increased over time [17]. The costs of breast cancer management, primarily driven by outpatient, emergency department (ED) visits and hospitalization, comprise the majority of overall medical costs $[18,19]$. Some studies suggested that the costs of anticancer therapies accounted for only $12 \%$ of total direct medical costs in metastatic BC in the US [20]. The findings reflected the clinical reality when traditional chemotherapies were primary systemic treatments for TNBC. The impact of emerging treatments (e.g., immunotherapies) on overall cancer care costs is unclear. BC also poses a considerable economic burden on patients due to high out-of-pocket costs and other expenditures relating to informal care [21]. Moreover, work loss and disability, particularly among working-age patients, add additional burden in young women [22]. Since TNBC commonly occurs at a younger age, the TNBC population is likely to experience an even greater economic burden. However, most publications on the cost of TNBC were conducted from the healthcare payer perspective. Research assessing the economic burden on patients, caregivers, and society was limited.

TNBC is associated with reduced health-related quality of life (HRQoL). Patients often experience distress because of fear of death or disease progression, a changed body image, and concern about loved ones [23, 24]. HRQoL is an important outcome to be considered when evaluating anticancer interventions. Previous studies have shown that with the availability of newer treatments and better cancer management, the quality of life of $\mathrm{BC}$ patients has improved in the last decade; however, pain, lymphedema, and anxiety are aspects of HRQoL that require further consideration [25]. Patients with TNBC are more likely to be associated with negative emotional states and worse HRQoL in comparison with non-TNBC subtypes. [26-28]

Although an increasing number of studies have been conducted assessing the HRQoL impact and cost burden posed by TNBC, to date these studies have not been reviewed systematically. A previous systematic literature review (SLR) by Parisi et al. [29] investigated the treatments, quality of life, and costs in metastatic HER2-negative breast cancer and TNBC with a literature search date of 2016; however, no studies of costs or quality of life in TNBC were included in this review. The objective of our study was to provide a systematic overview of the published literature and provide insights into the economic and humanistic burden of TNBC to assist medical decisions for healthcare payers, providers, and patients. 


\section{Methods}

\subsection{Literature Review}

A systematic review was performed in accordance with the Preferred Reporting Items for Systematic Reviews and MetaAnalyses (PRISMA) guidelines to identify publications that describe the economic or humanistic burden of TNBC [30]. A comprehensive literature search was conducted in Excerpta Medica dataBASE (EMBASE), Medical Literature Analysis and Retrieval System Online (MEDLINE), Econlit, the Cochrane Central Register of Controlled Trials (CENTRAL), and the Cochrane Database of Systematic Reviews. The search included studies published from database inception to 16 May 2021. Additionally, a targeted search was conducted in the Northern Light Life Sciences Conference Abstracts database for the following conference proceedings from 2016 through June 2021: the American Society of Clinical Oncology Annual Meeting (2016-2020); the European Society for Medical Oncology Congress (2016-2020); the International Society for Pharmacoeconomics and Outcomes Research Annual European Congress (2016-2020), the International Society for Pharmacoeconomics and Outcomes Research Asia-Pacific Conference (2016-2021), the International Society for Pharmacoeconomics and Outcomes Research Annual International Meeting North America (2016-2021), the National Comprehensive Cancer Network Annual Conference (2016-2020), and the San Antonio Breast Cancer Symposium (2016-2020). In addition, the bibliographies of any relevant articles were reviewed for any studies potentially not captured by the databases. Only articles published in English were reviewed due to resource challenges with respect to expertise in non-English languages. Detailed search strategies are presented in electronic supplementary Tables S1-S7.

The eligibility criteria for study inclusion are summarized in Table 1. The search strategy included studies in earlystage, locally advanced non-mTNBC and mTNBC. This review was limited to TNBC, but for studies that included TNBC with other BC types, subgroup analyses for the relevant patients from these studies were included in the review. Eligible study designs included economic evaluations, observational studies, and clinical trials. All abstracts identified from bibliographic databases were screened for study eligibility. Abstracts deemed eligible were screened again by viewing the complete study publication to make a final determination as to whether they met the population, intervention, comparison, outcomes, and study design (PICOS) criteria.

\subsection{Study Selection and Data Extraction}

The study screening and selection process are summarized using a PRISMA flow diagram. [30] Only studies that had primary data on the economic or humanistic burden of TNBC were selected such that previously published information was not repeated. Cost-effectiveness studies were excluded because none of them were primary studies on costs or HRQoL. They typically derived outcomes based on simulation modeling using data reported from other studies. Study screening and data extraction were performed by two individuals working independently. These individuals compared their completed work to identify discrepancies and resolve them through consensus, including a third individual if needed.

Data were obtained from real-world studies, clinical trials and economic evaluations. For the economic burden of TNBC, data were extracted for healthcare resource utilization (HCRU), direct costs, and indirect costs. For the humanistic burden of TNBC, data were extracted for the following generic patient-reported HRQoL measures: EuroQol-5 Dimension (EQ-5D), Health Utilities Index Mark-2 (HUI-2), HUI-3, Short-Form Six-Dimension (SF-6D), Short Form 36 Health Survey Questionnaire (SF-36), European Organization for Research and Treatment of Cancer Quality-of-Life Questionnaire-Core 30 (EORTC QLQ-C30), Patient-Reported Outcome Measurement Information System Fatigue-Short Form (PROMIS F-SF-1), Qualityadjusted Time Without Symptoms of Disease Progression and Toxicity (Q-TWIST), Cancer Therapy Satisfaction Questionnaire (CTSQ), etc., and disease-specific qualityof-life measures: EORTC QLQ-Breast Cancer Module 23 (BR23), Functional Assessment of Cancer Therapy Breast Symptom Index (FACT-B), Functional Assessment of Cancer Therapy-General (FACT-G), and utility measures.

\subsection{Quality Assessment}

Two different tools were used to assess the quality of the included studies. The Cochrane Collaboration Risk of Bias Tool [31] was used to evaluate the randomized controlled trials (RCTs). The tool assesses risk of bias of studies as a judgment (high, low, or unclear risk) for individual elements from six domains (selection, performance, detection, attrition, reporting, and other). The Newcastle-Ottawa Quality Assessment Scale (NOS) [32], a 9-star system including three broad perspectives (selection, comparability, and outcome), was used to assess the quality of non-randomized trials and cohort studies. Scores of 0-3, 4-6, 7-9 were considered as low, moderate, and high quality, respectively. An adapted version of the NOS [32] was used for cross-sectional studies. The quality assessment was conducted by two independent reviewers. Following reconciliation between the 
Table 1 Eligibility criteria for study inclusion

\begin{tabular}{llc}
\hline Criteria & Economic burden studies & Humanistic burden studies \\
\hline Population & Early-stage, locally advanced, non-metastatic TNBC & \\
& Metastatic TNBC & \\
Interventions & Not restricted & \\
Comparators & Not restricted & Generic patient-reported outcomes measures (EQ-5D, HUI-2, \\
Outcomes & Direct costs & HUI-3, SF-6D, SF-36, EORTC QLQ-C30, PROMIS-Fatigue \\
& Indirect costs & SF1, Q-TWIST, CTSQ, etc.) \\
& Healthcare resource utilization & Disease-specific health-related quality of life (EORTC QLQ- \\
& & BR23, FACT-B, FACT-G) \\
& & Utility measures \\
Study design & Observational studies (e.g., prospective and retrospective cohort studies, case-control studies, cross-sectional studies, con- \\
& trolled and uncontrolled longitudinal studies) & \\
& Randomized controlled trials and non-randomized clinical trials & \\
& Economic evaluations (e.g., cost-effectiveness analyses, budget impact analyses, and cost-of-illness analyses) \\
& Literature reviews summarizing the results of primary research studies and/or economic evaluations ${ }^{\mathrm{a}}$ \\
\hline
\end{tabular}

CTSQ Cancer Therapy Satisfaction Questionnaire, EORTC QLQ-BR23 European Organization for Research and Treatment of Cancer Qualityof-Life Questionnaire - Breast Cancer Module 23, EORTC QLQ-C30 European Organization for Research and Treatment of Cancer Quality-ofLife Questionnaire-Core 30, EQ-5D EuroQol-5 Dimension, FACT-B Functional Assessment of Cancer Therapy - Breast Symptom Index, FACT$G$ Functional Assessment of Cancer Therapy - General, HUI-2 Health Utilities Index Mark-2, HUI-3 Health Utilities Index Mark-3, PROMIS Fatigue SF-1 Patient-Reported Outcome Measurement Information System Fatigue-Short Form, SF-6D Short-Form Six-Dimension, SF-36 Short-Form 36 Health Survey Questionnaire, SLRs systematic literature reviews, TNBC triple-negative breast cancer, $Q-T W I S T$ Quality-Adjusted Time Without Symptoms of Disease Progression and Toxicity

${ }^{a}$ Literature reviews that involve some methodology for study identification and study selection were of interest for the purposes of cross-referencing. This included SLRs, scoping reviews, and landscape reviews. Narrative reviews that did not involve study identification via databases and that are primarily summarizing the author's viewpoints are not of interest

two reviewers, a third investigator was included to reach a consensus for any remaining discrepancies.

\subsection{Cost Adjustment}

To facilitate comparisons across studies from varying time periods and country settings, costs were adjusted to 2021 US dollars (US\$) using the methods outlined by Turner et al. [33] All studies were conducted in developed countries; thus, costs were first converted to US\$ using the exchange rate during the cost year and then inflated using the Consumer Price Index. [34] When the cost year was not reported, the year of publication represented the cost year. In this review, adjusted costs in 2021 US $\$$ and the original costs are both reported in summary tables.

\section{Results}

The selection process to identify studies that evaluate the economic and humanistic burden of TNBC are presented as PRISMA flow diagrams in Figs. 1 and 2. The searches resulted in the identification of 1505 and 1364 citations accessing the economic and humanistic burden, respectively. After abstract screening, 39 full-text articles were assessed for eligibility, and a total of 19 studies meeting the PICOS criteria were included in the economic review. Sixty full-text articles were assessed for eligibility and 10 studies meeting the PICOS criteria were included in the humanistic burden review. A list of all studies included in the review is presented in Table 2.

\subsection{Economic Burden of Triple-Negative Breast Cancer (TNBC)}

Table 2a displays the characteristics of the 19 studies published between 2012 and 2021 that assessed direct medical costs, HCRU, and indirect costs associated with TNBC. Ten studies included in the review assessed both the direct costs and HCRU [35-44]. Six studies only assessed the direct costs [41, 45-49], while two studies only evaluated HCRU associated with TNBC [50, 51]. Two studies assessed both direct and indirect costs of TNBC [45, 52], although one study reported indirect costs only [53]. Thirteen studies were conducted in the US, and one study each was conducted in Canada, Spain, Portugal, France, Belgium, and Sweden.

\subsubsection{Study Quality Assessment}

The methodological quality of the 18 cohort studies was assessed using the NOS. Twelve studies were rated as high quality and six studies were rated as moderate quality. No studies were categorized as low quality. The studies with moderate quality usually failed to provide details on study 
Fig. 1 Study selection for economic burden studies

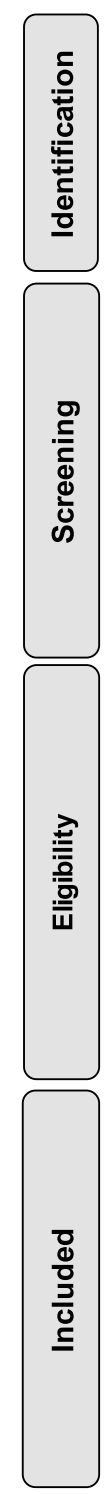

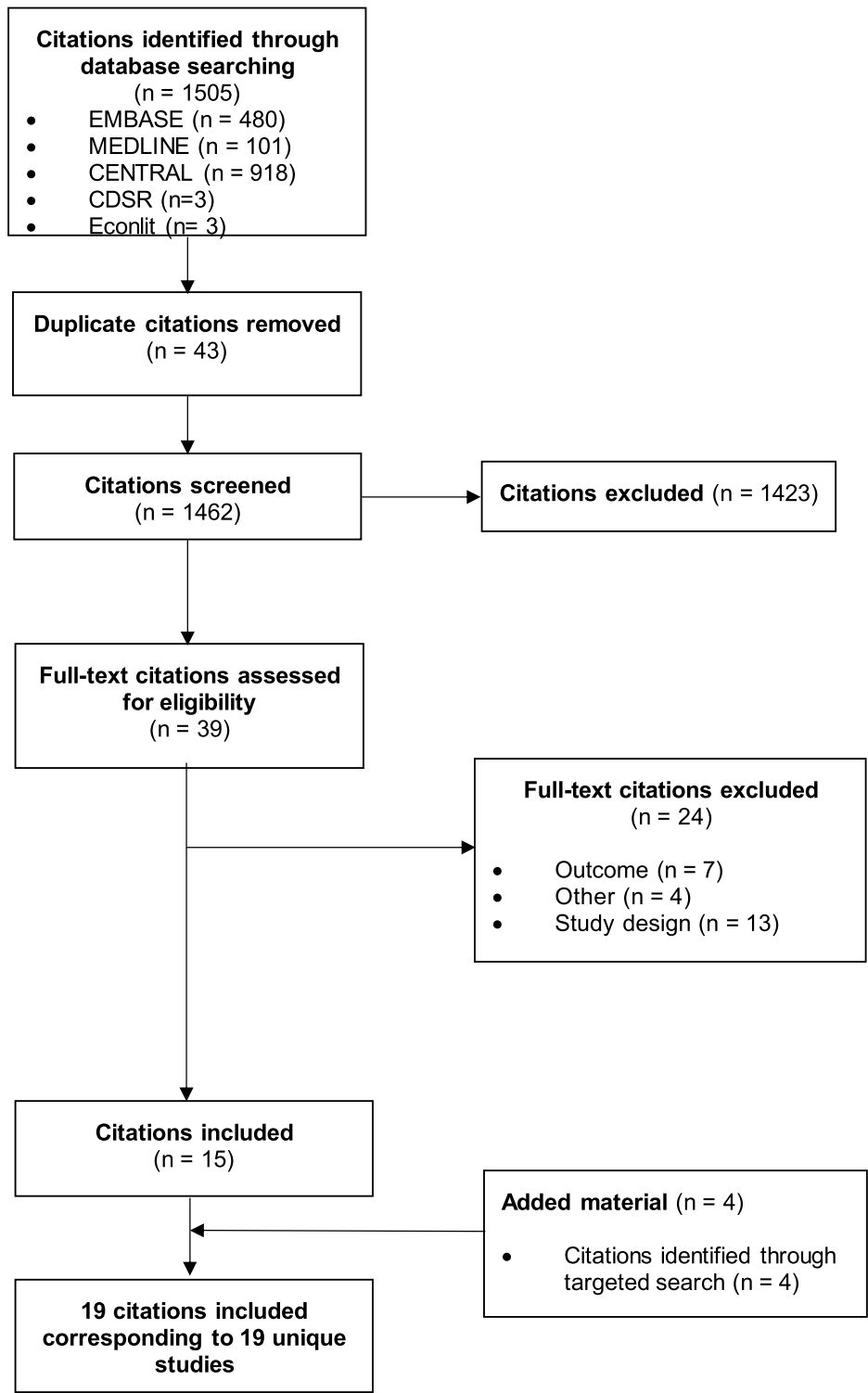

follow-up periods or comparability of the cohorts. Formal quality assessment was not conducted for the study by De las Heras et al. [45] because the study adopted a cost-of-illness modeling design and the risk of bias assessment tools do not apply.

\subsubsection{Direct Costs and Healthcare Resource Utilization}

Studies evaluating direct costs and HCRU varied widely in study setting, time horizon, patient populations, and treatment patterns. Study country, perspective, and setting are listed in Table $2 \mathrm{a}$. Table 3 summarizes direct medical costs by patient characteristics (including population size, age, and cancer severity range of the study population), study time horizon, subpopulations, treatment periods, base cost year, currency, and cost elements evaluated. A summary of hospitalization and ED resource utilization is presented in Table 4. Cancer severity was reported by stage for many cost studies, defined as non-recurrence, locoregional recurrence, and distant recurrence (metastases), or stage I, II, III, and IV, with stage IV (mTNBC) being the most severe. The treatment period was often categorized as the neoadjuvant and adjuvant period for eTNBC (stage I-IIII) and by line of therapy (first, second, third or late line) for mTNBC. The lifetime (or other time horizon) per-patient costs were estimated in one US study and four ex-US studies (Table 3a), while most studies focused on per-patient unit time (e.g., per month) costs (Table $3 \mathrm{~b}$ ). The studies were published between 2012 and 2021 and assumed a base cost year ranging from 2010 to 2020. 
Fig. 2 Study selection for humanistic burden studies
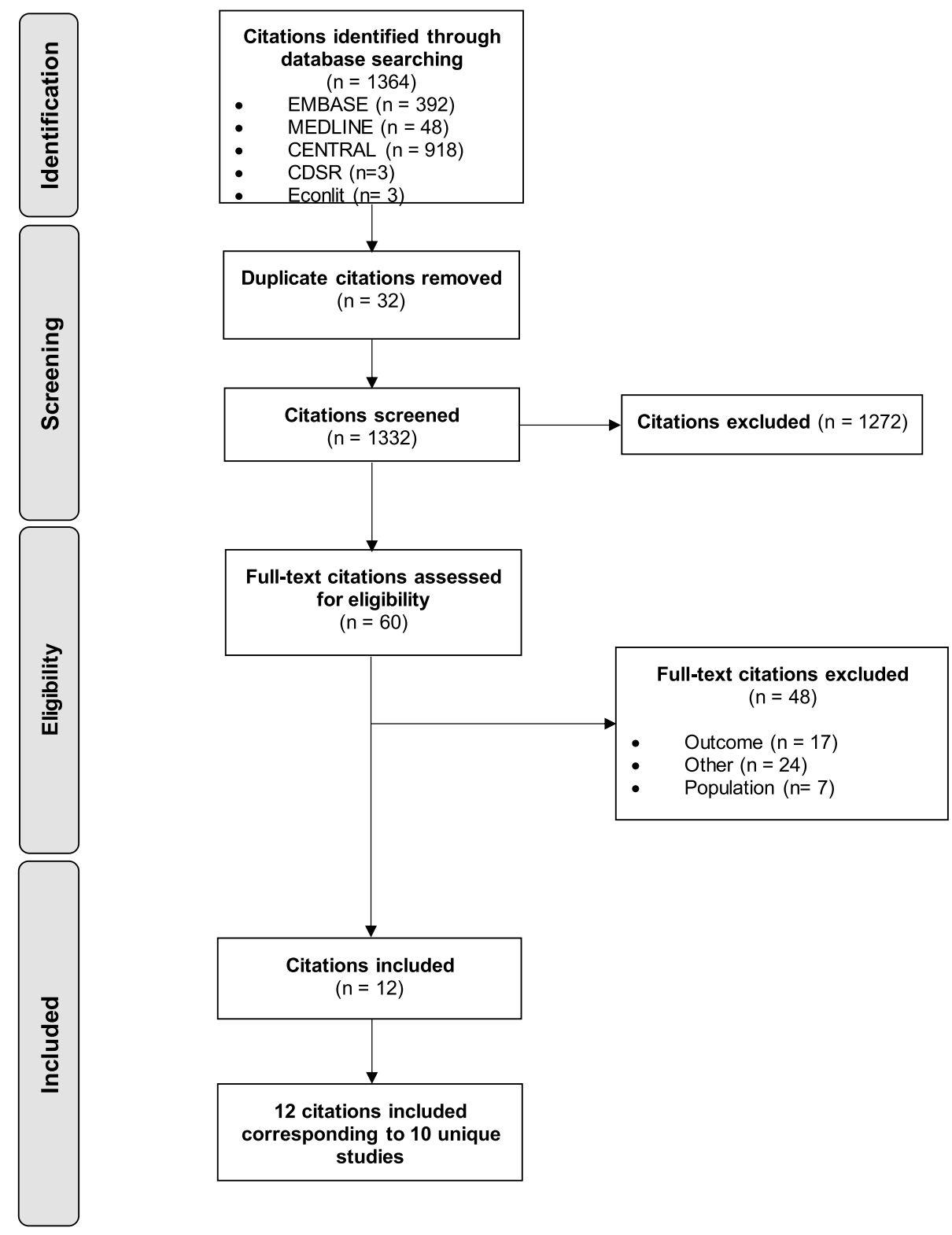

Healthcare System Burden Studies showed that TNBC was more resource-intensive and more costly to treat compared with other BC subtypes. For example, Baser et al. $[36,37]$ examined health outcomes and healthcare costs for women with TNBC compared with non-TNBC in two retrospective studies using a US managed care registry. The studies showed that patients with TNBC had significantly shorter survival, accompanied by greater inpatient and ED utilization and higher healthcare costs. Other studies (Houts et al. [38], Brandão et al. [46], and Parikh et al. [50]) also reported more evident unmet need, poorer health outcomes, and higher cost of care among TNBC patients than their non-TNBC counterparts.

Aly et al. [35] estimated the lifetime direct medical costs to be $\$ 83,033$ per patient for patients diagnosed with
mTNBC, from the US Medicare perspective. The total cost of care for mTNBC was also reported in a Spanish study (De las Heras et al. [45]) over 5 years following metastatic disease diagnosis $(\$ 118,635)$ and a French study (Mery et al. [47]) for mTNBC patients who received bevacizumab plus paclitaxel as first-line treatment $(\$ 32,106)$. Brandão et al. [46] evaluated direct medical costs for eTNBC (stage I-III) care in a Portuguese cancer center setting and reported a median cost of $\$ 13,346$ per patient over the first 3 years after diagnosis. Similarly, total treatment costs of eTNBC were evaluated (by Roman et al. [48]) at a breast clinic in Belgium $(\$ 28,003)$. All costs reported above were adjusted to 2021 US\$. Cost estimates vary widely across studies and are difficult to compare due to substantial heterogeneity in study 


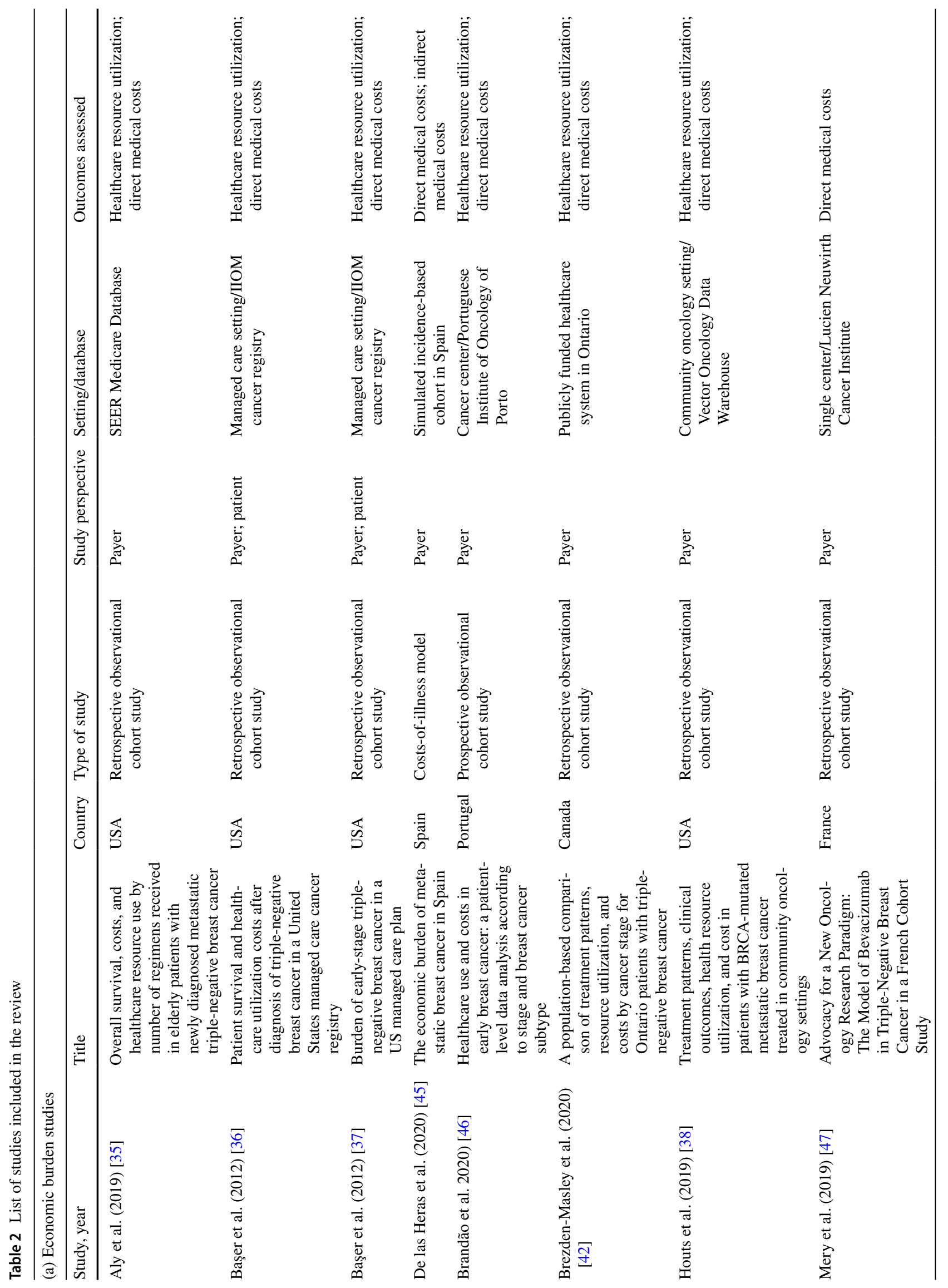




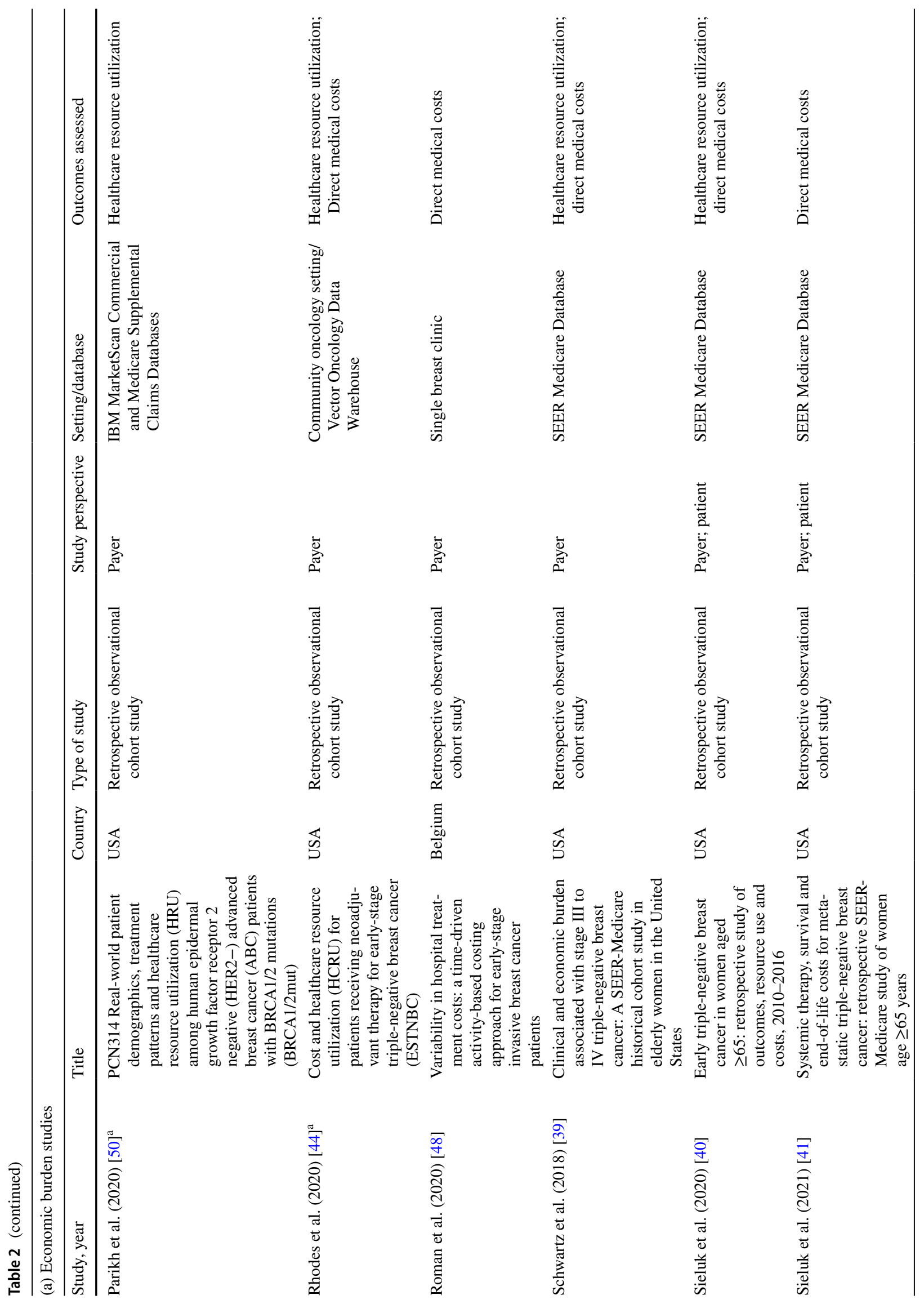




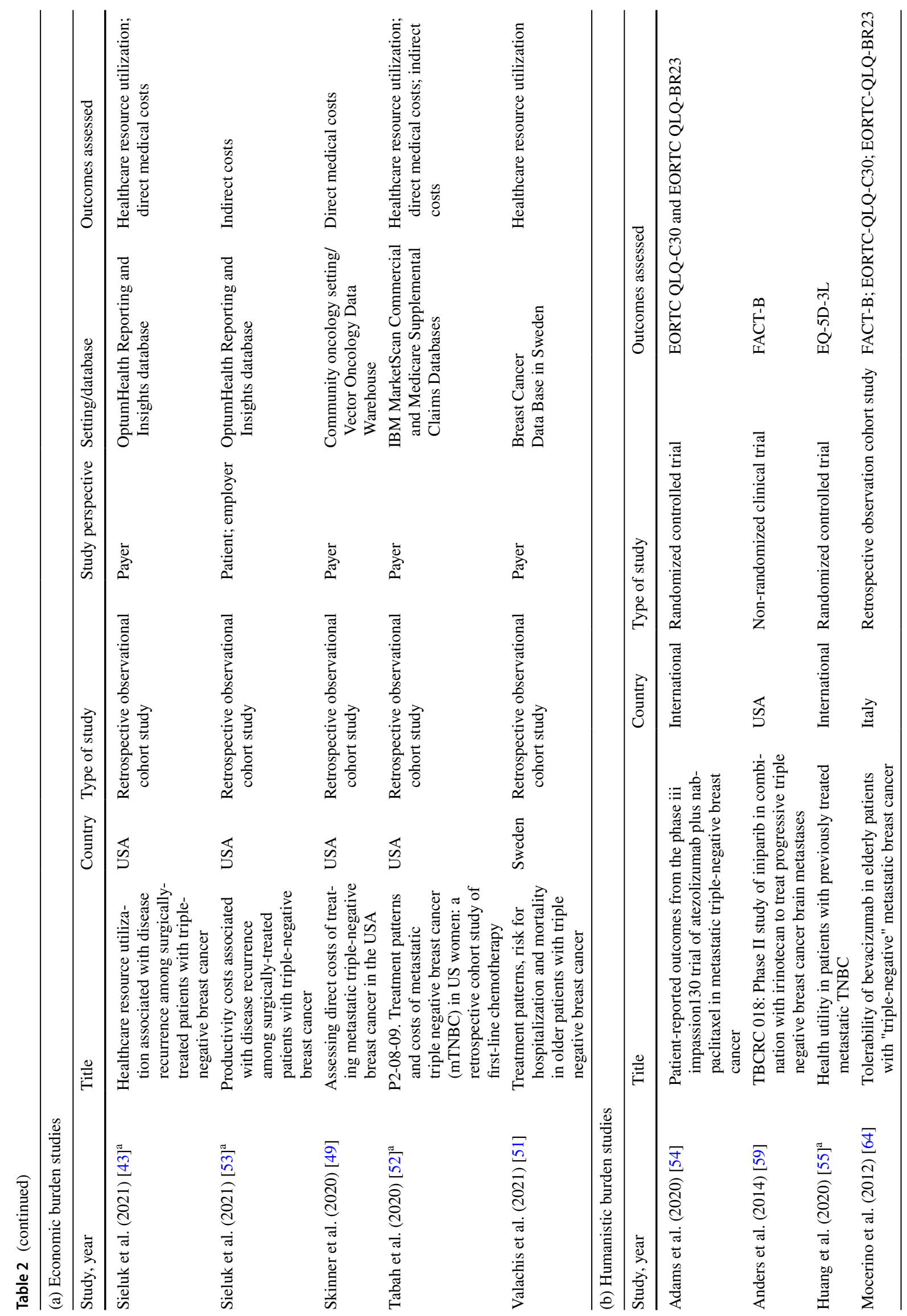




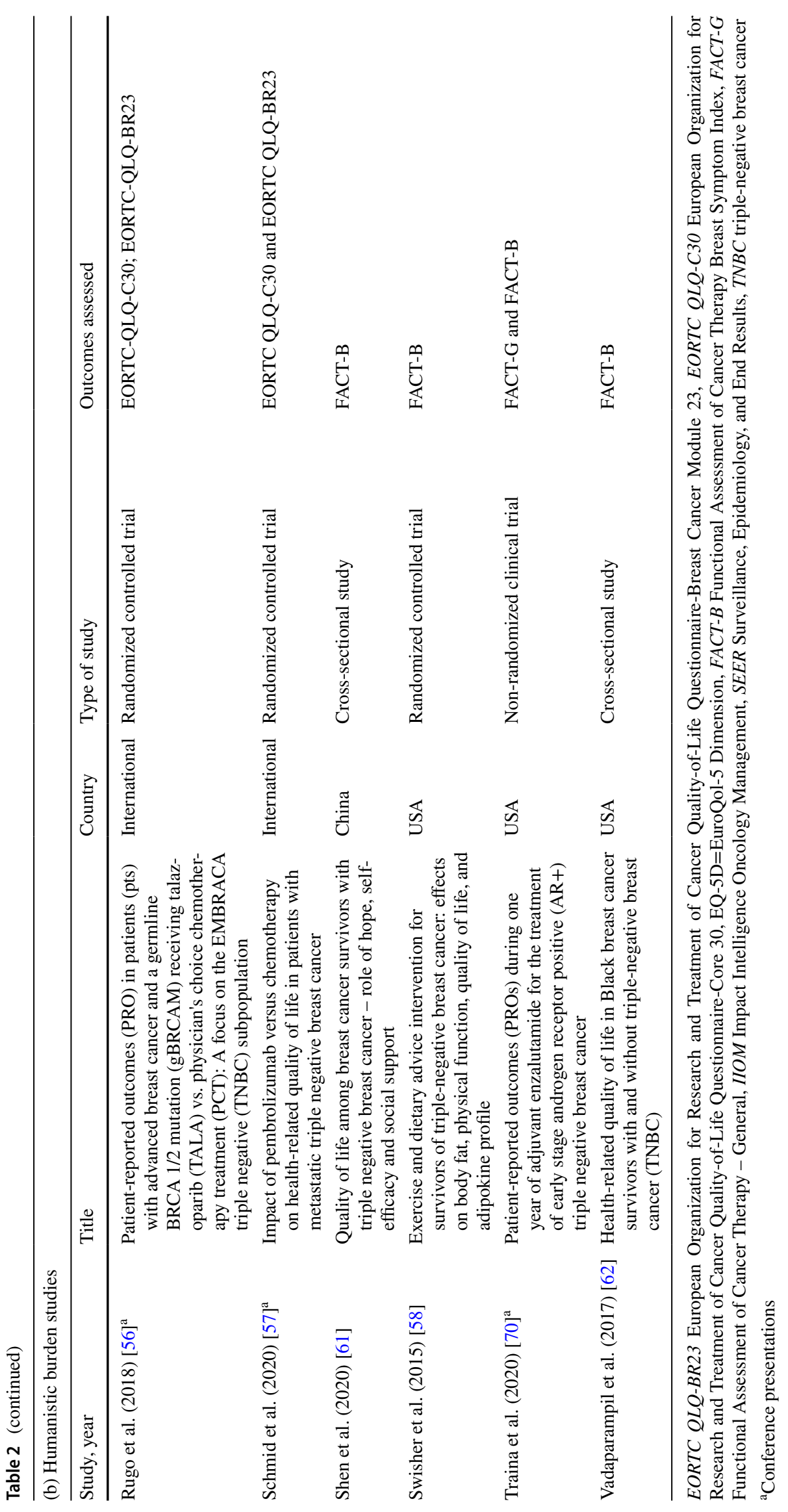




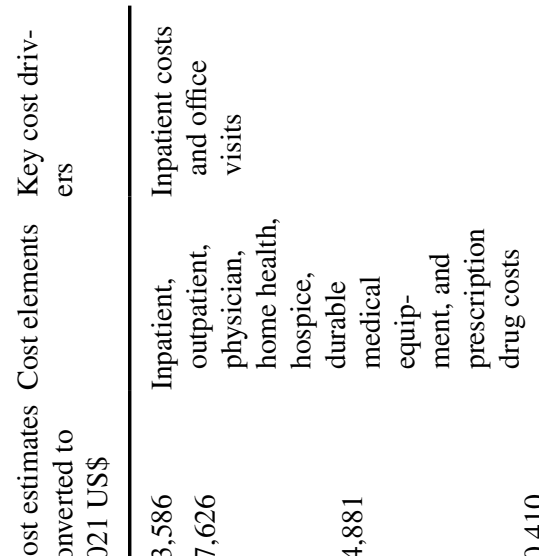

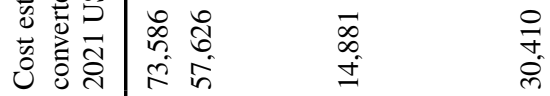

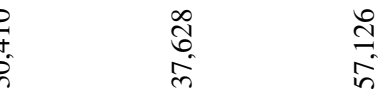

0
0
0
0
0
0
0
0
0
0
0
0
0
0
0
0

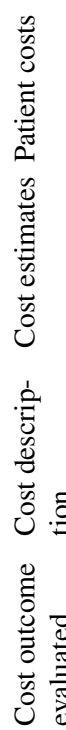

출

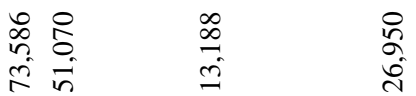

ले

$\begin{array}{ll}\text { fे } & \widehat{c} \\ m & \stackrel{0}{n}\end{array}$

宫

¿.

落

$\sum_{\Sigma}^{\infty}$

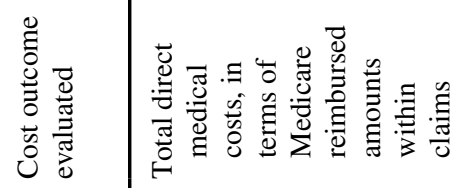

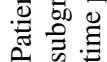

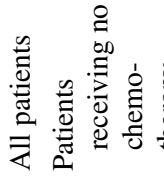

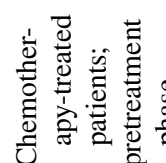

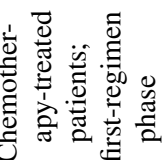

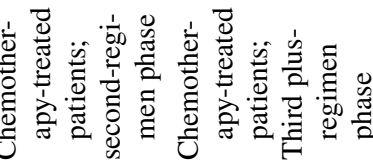

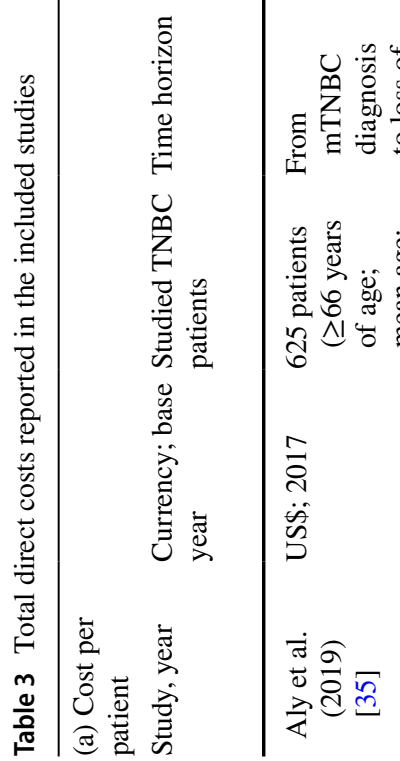




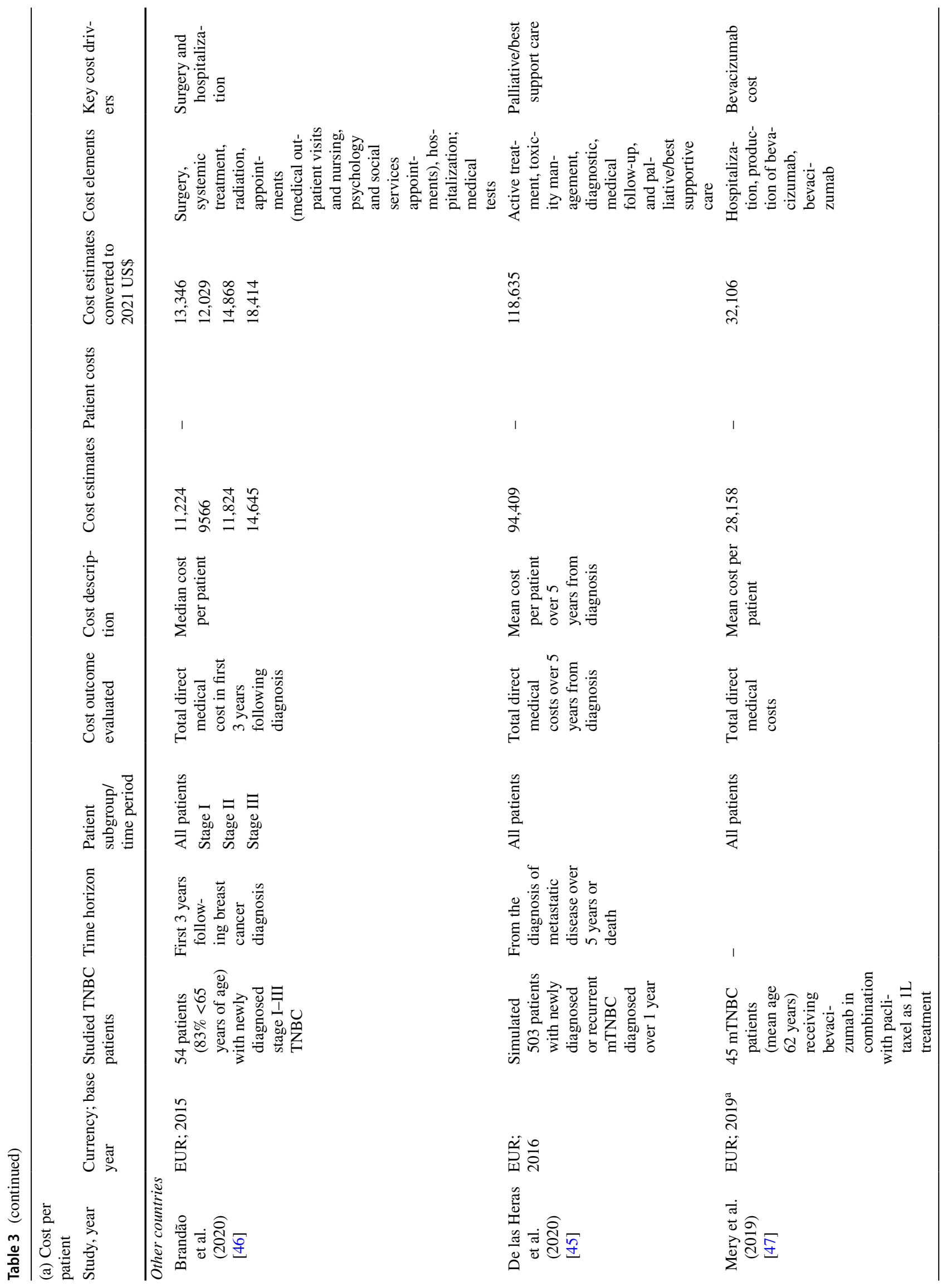




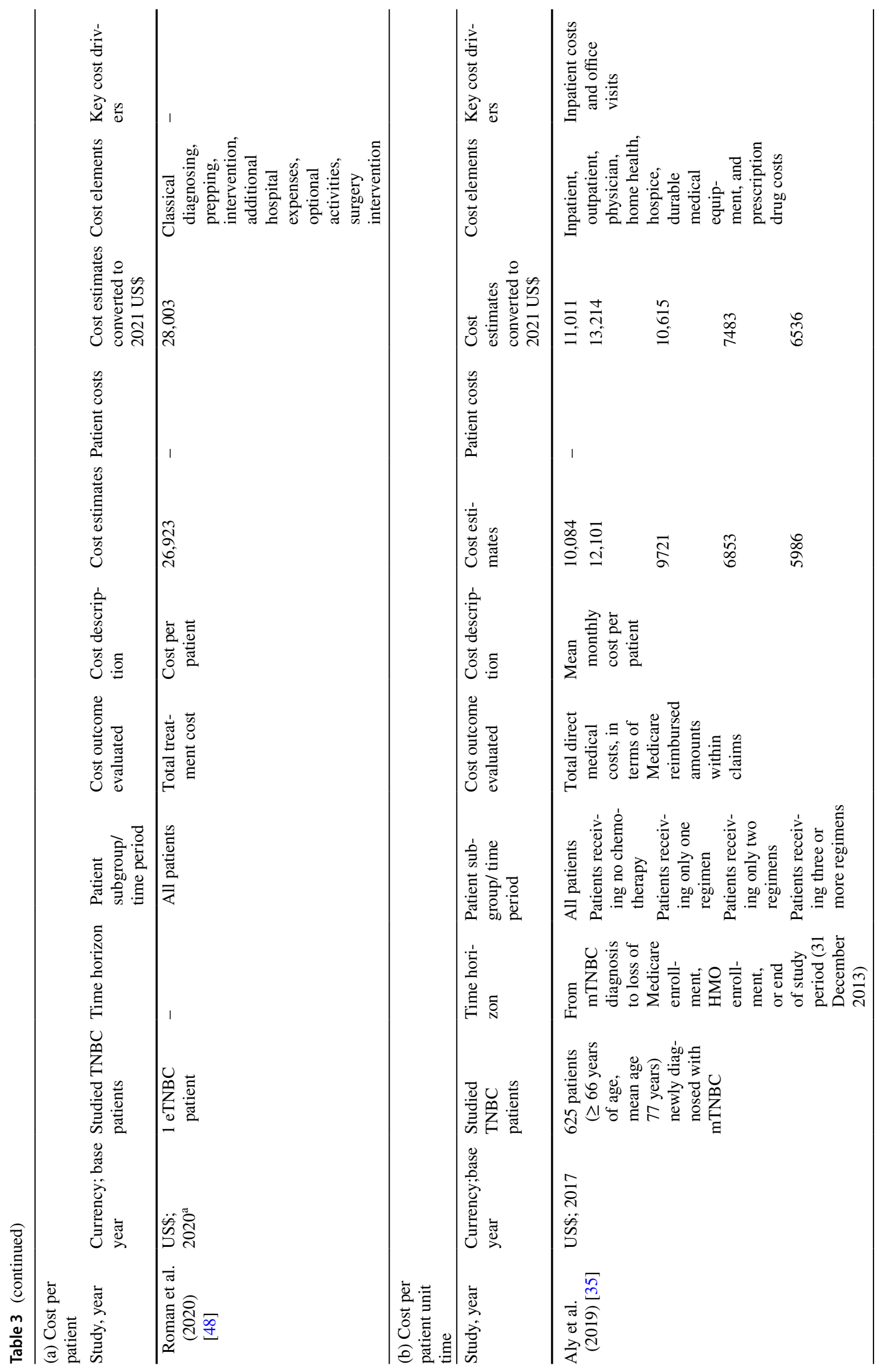




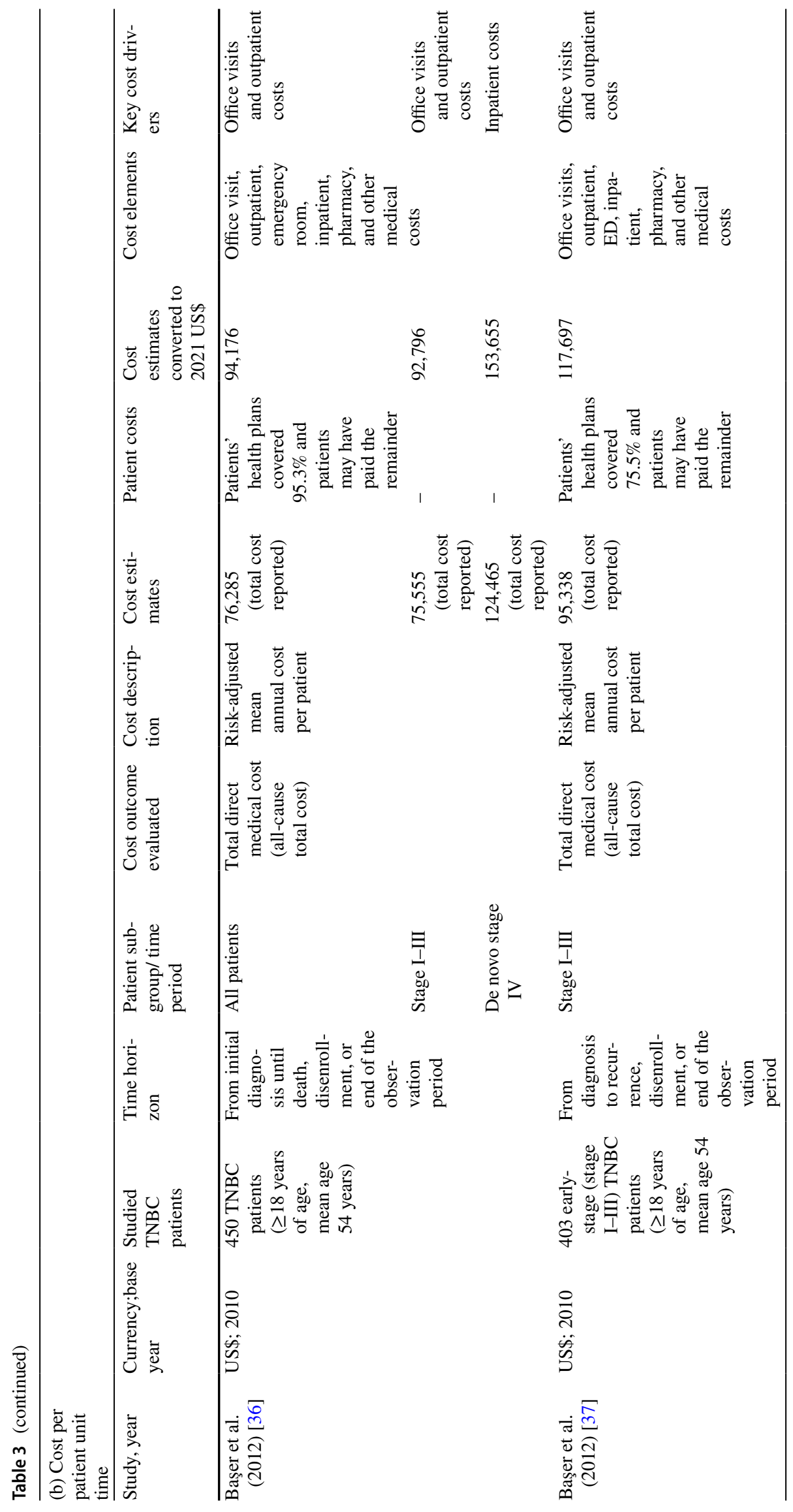




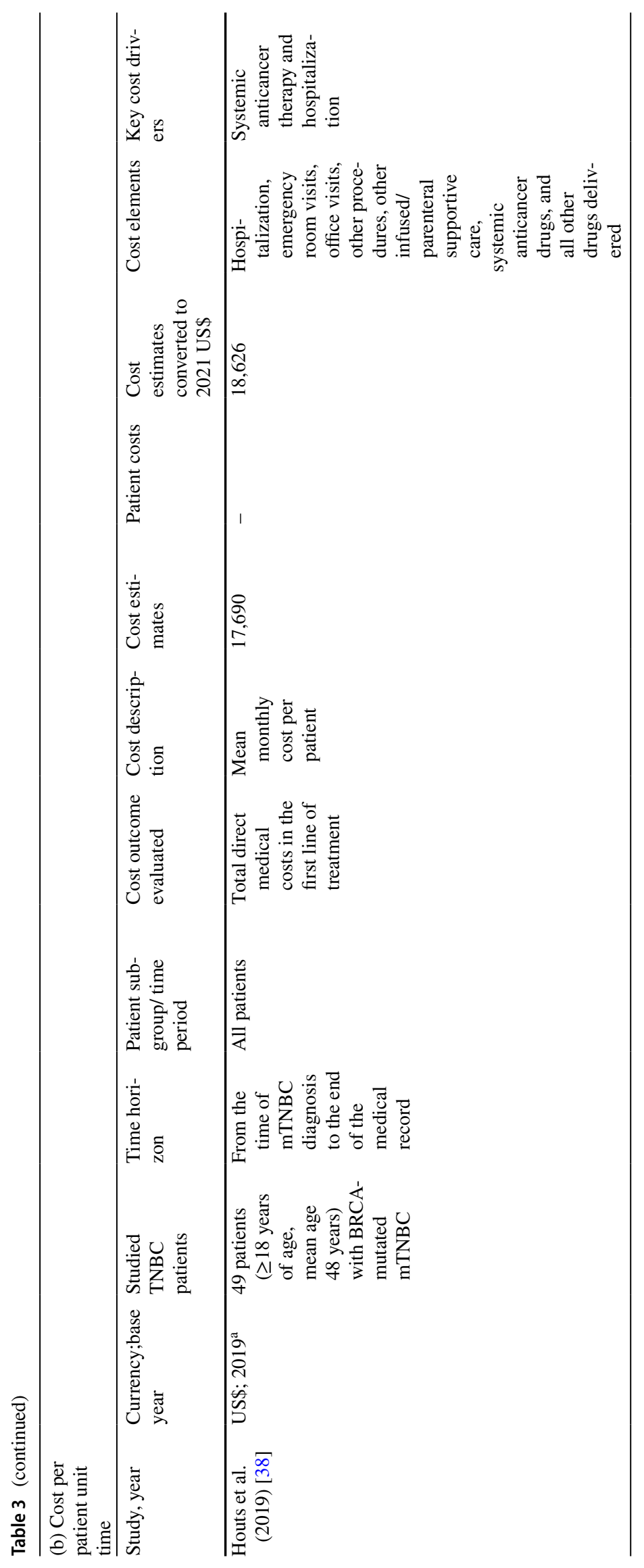




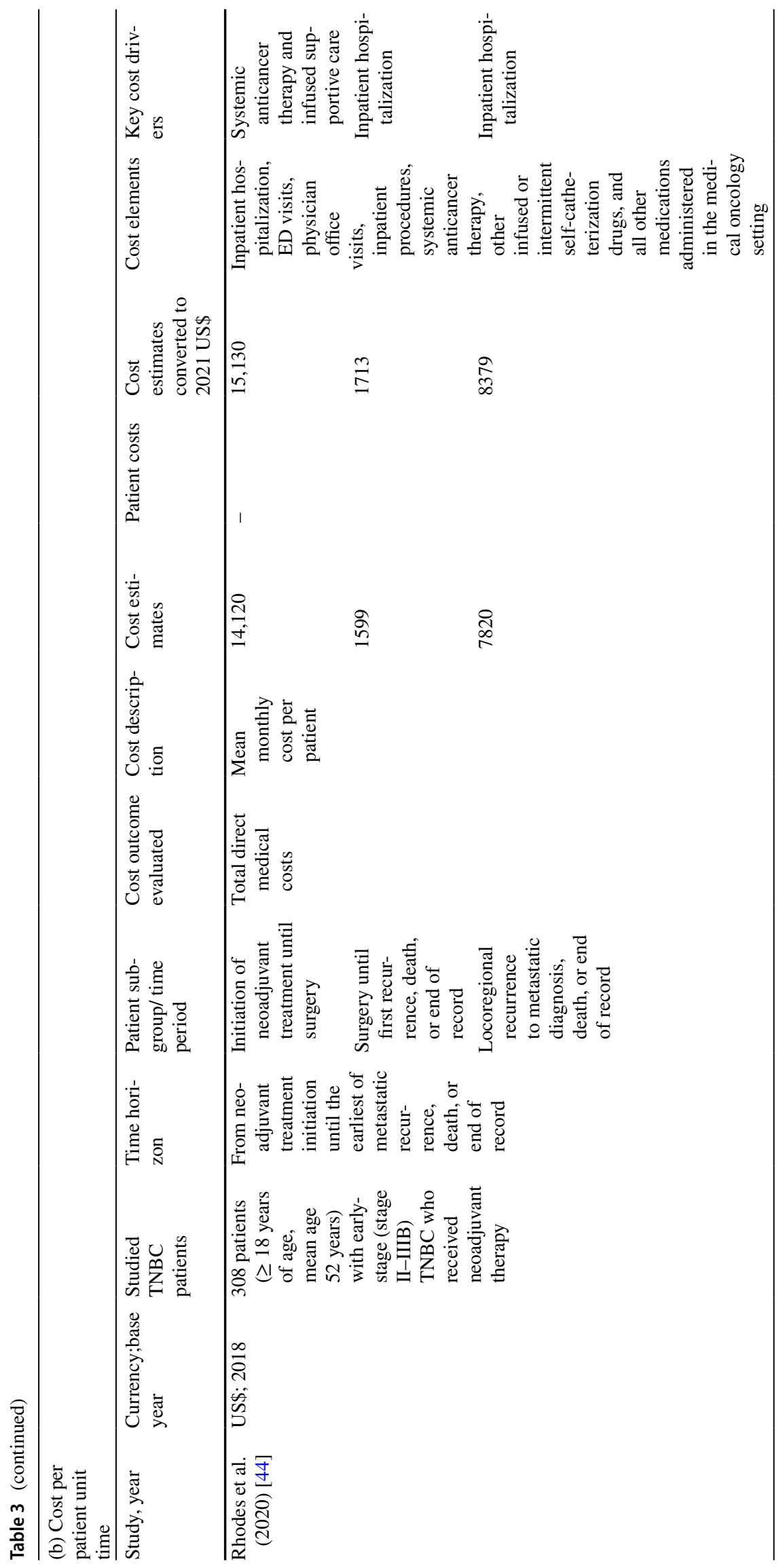




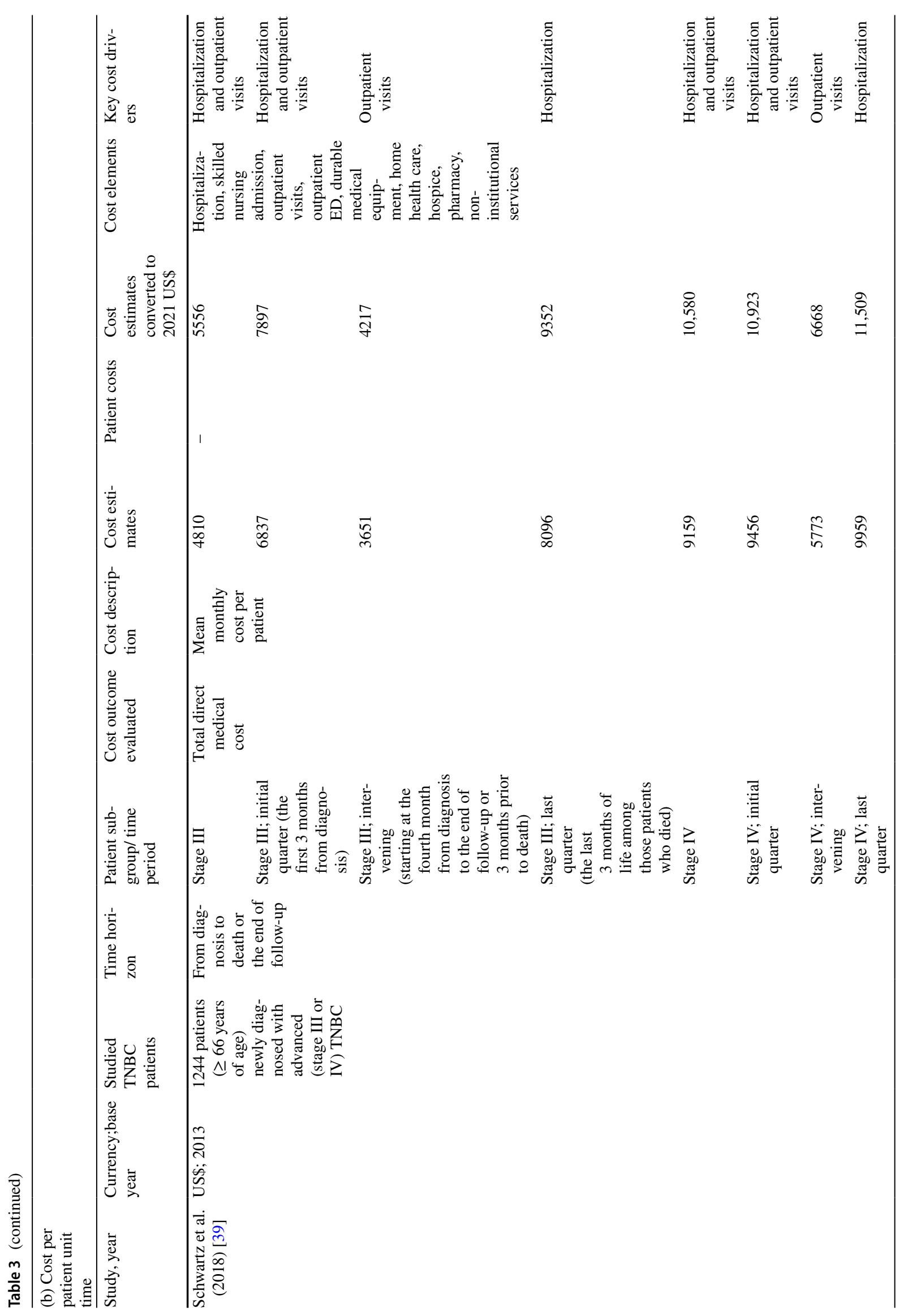




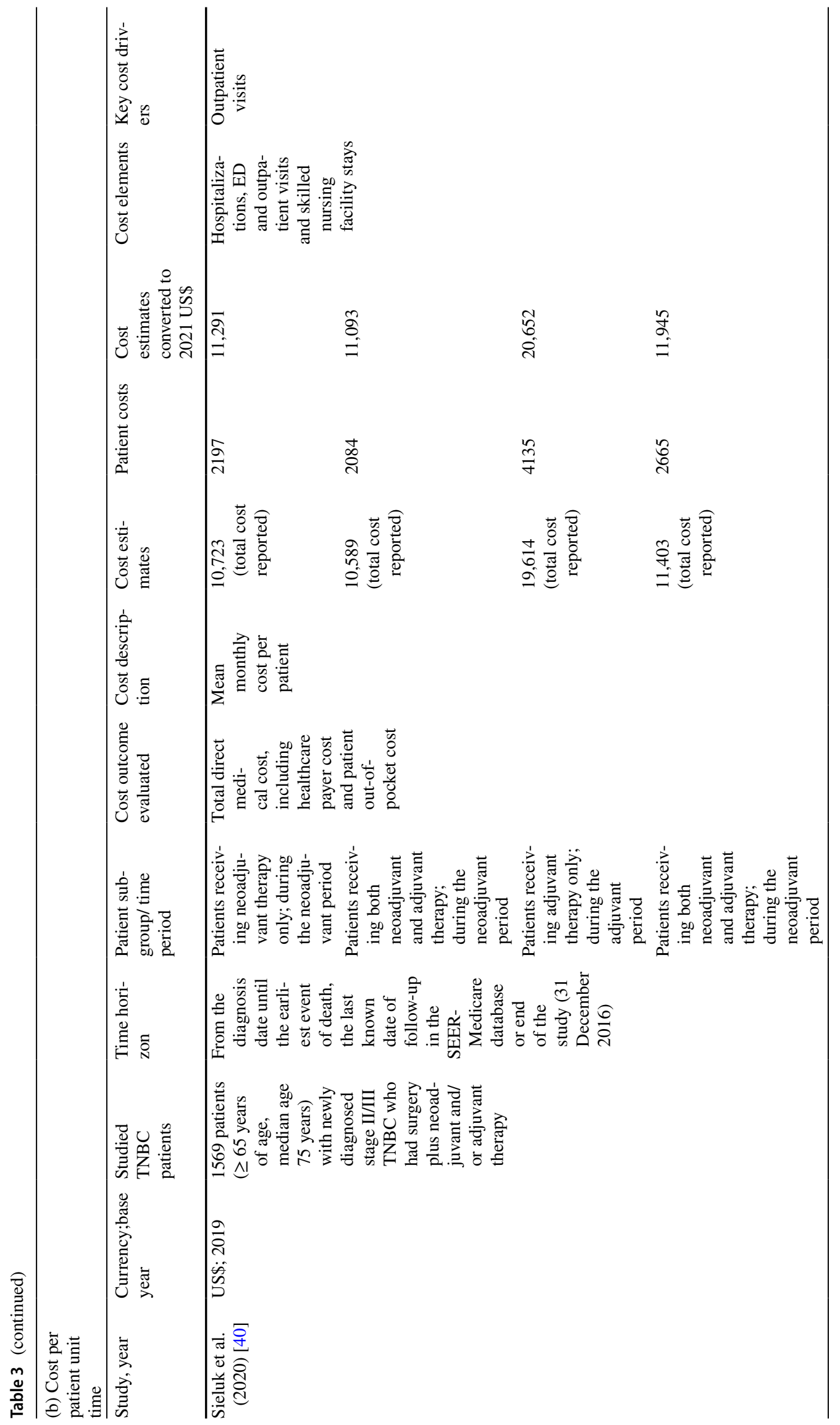




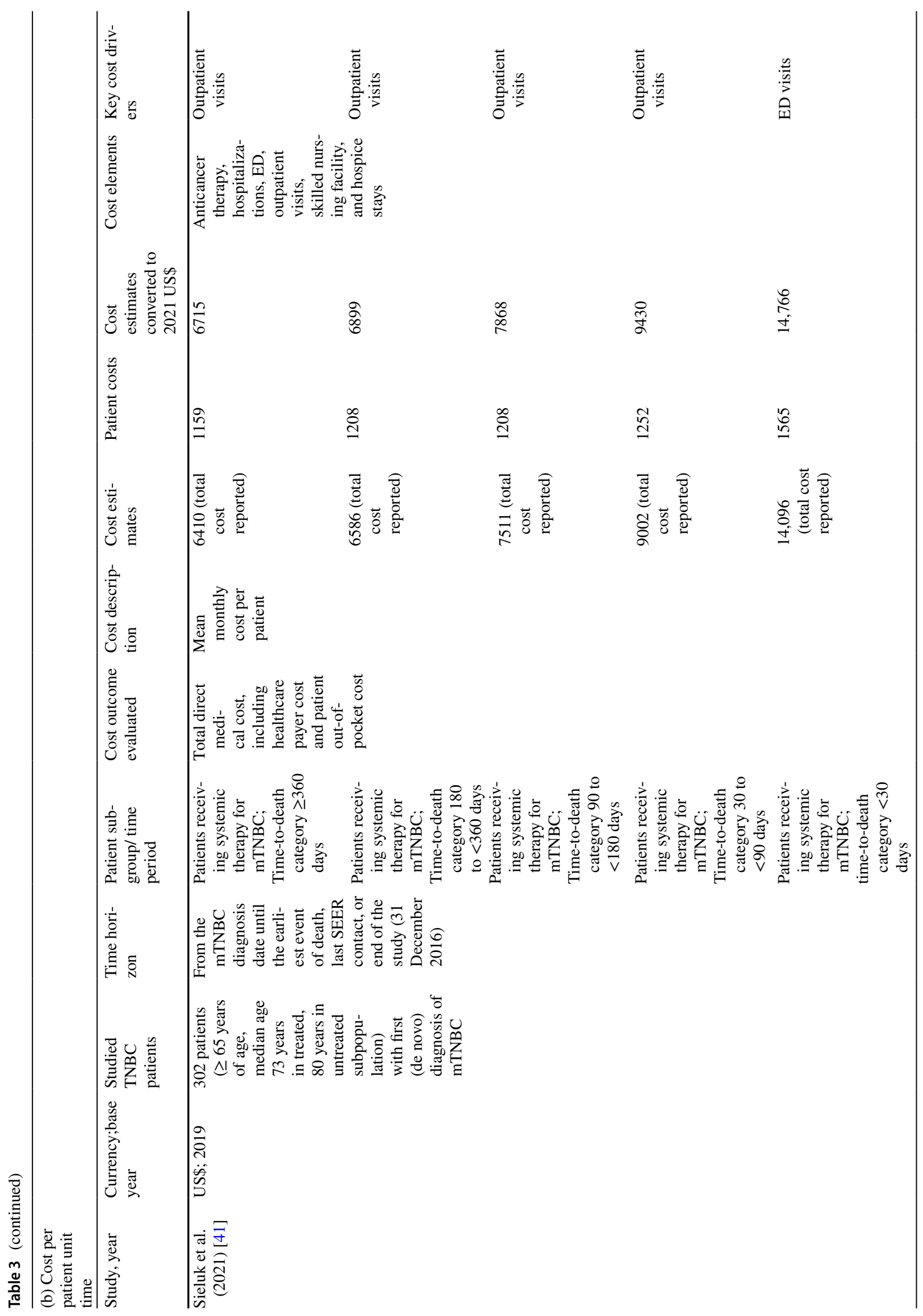




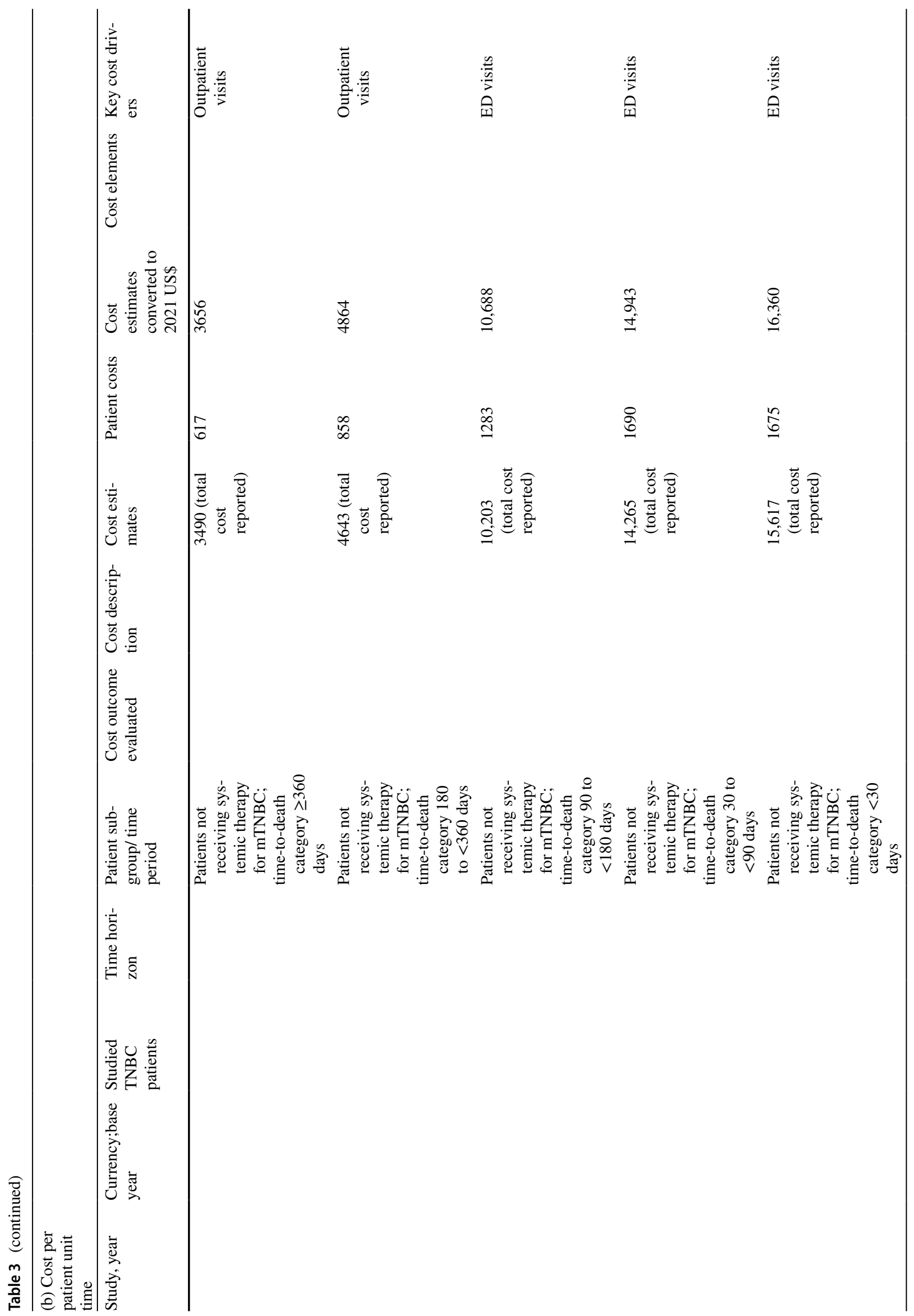




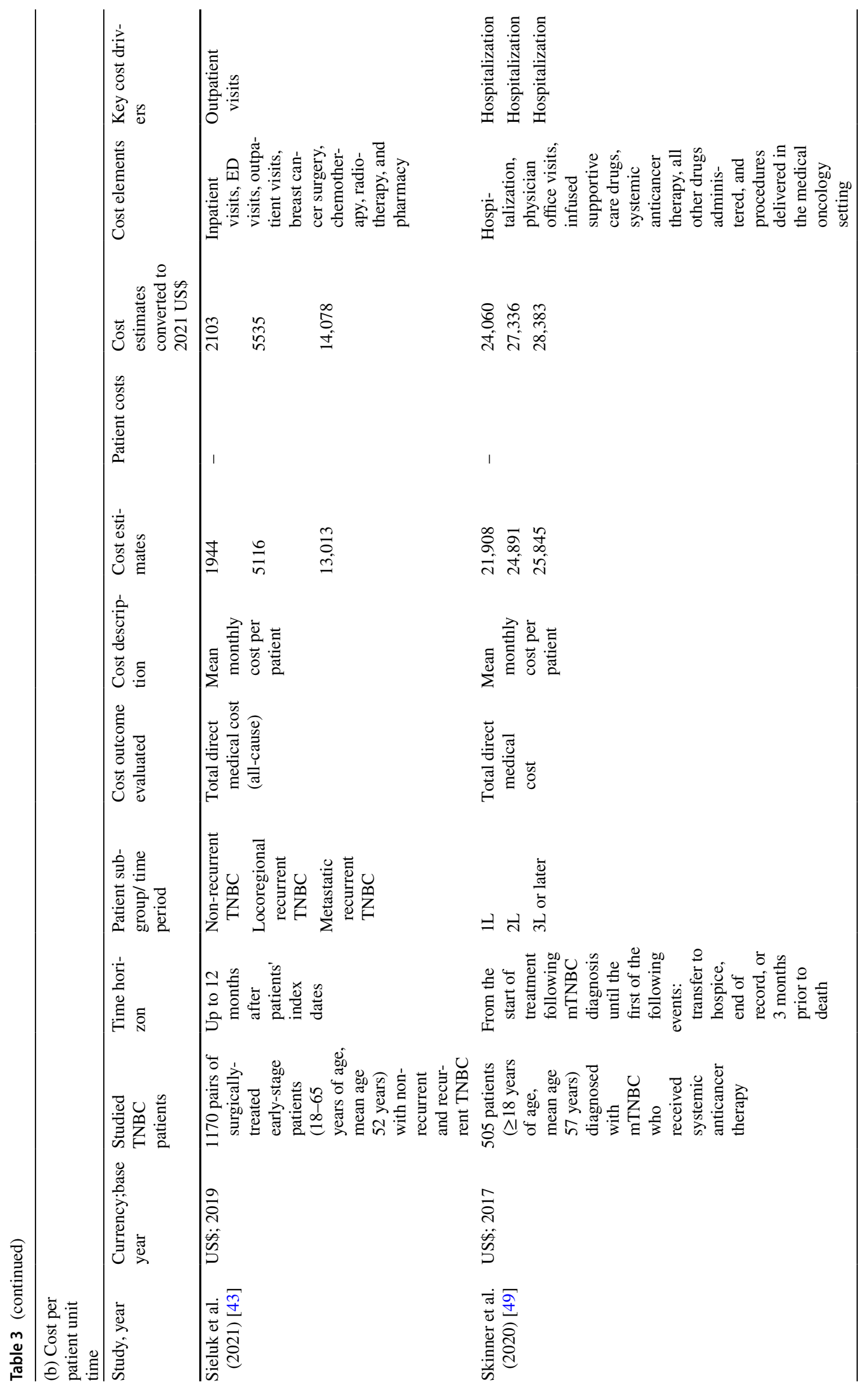




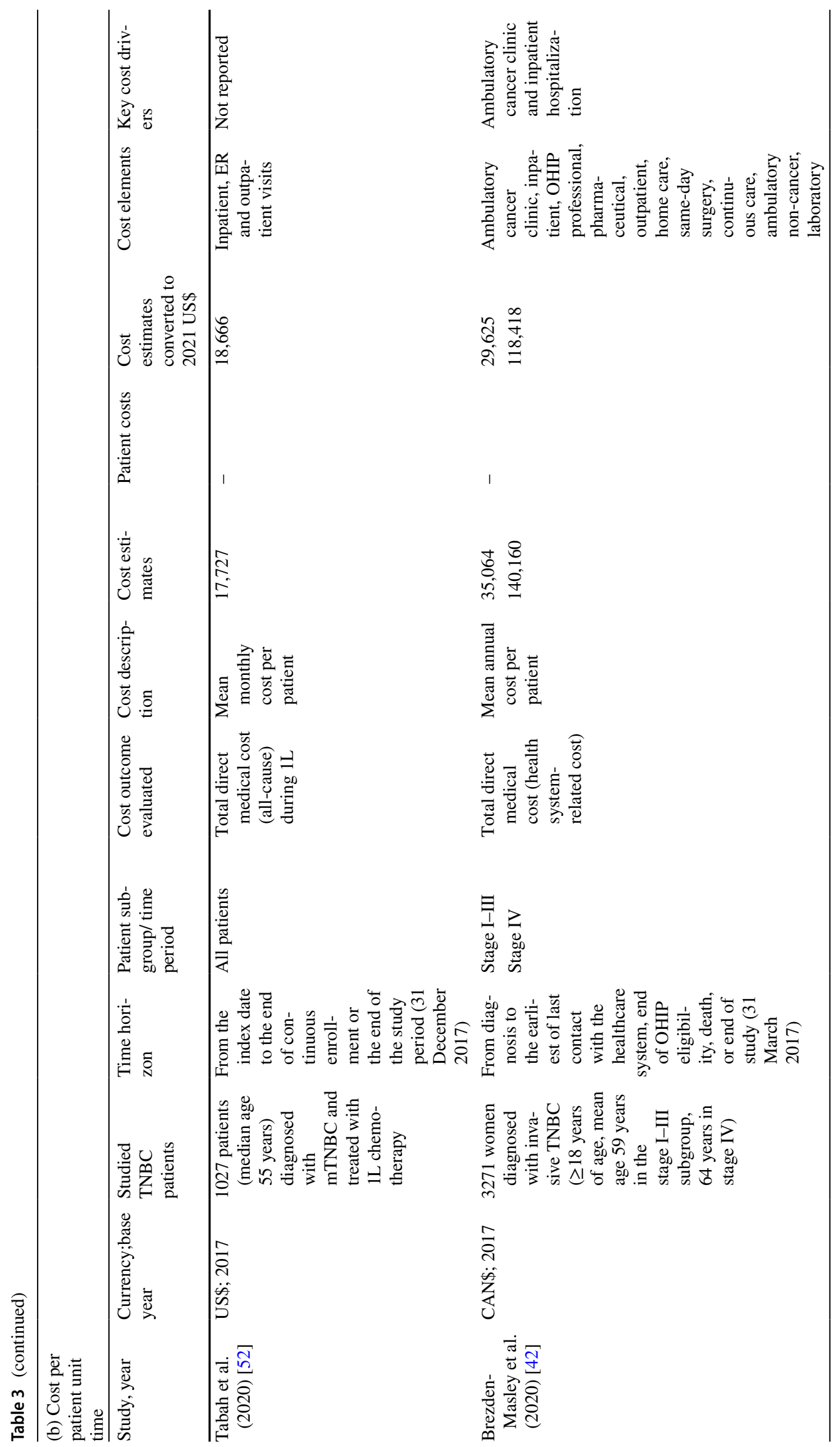




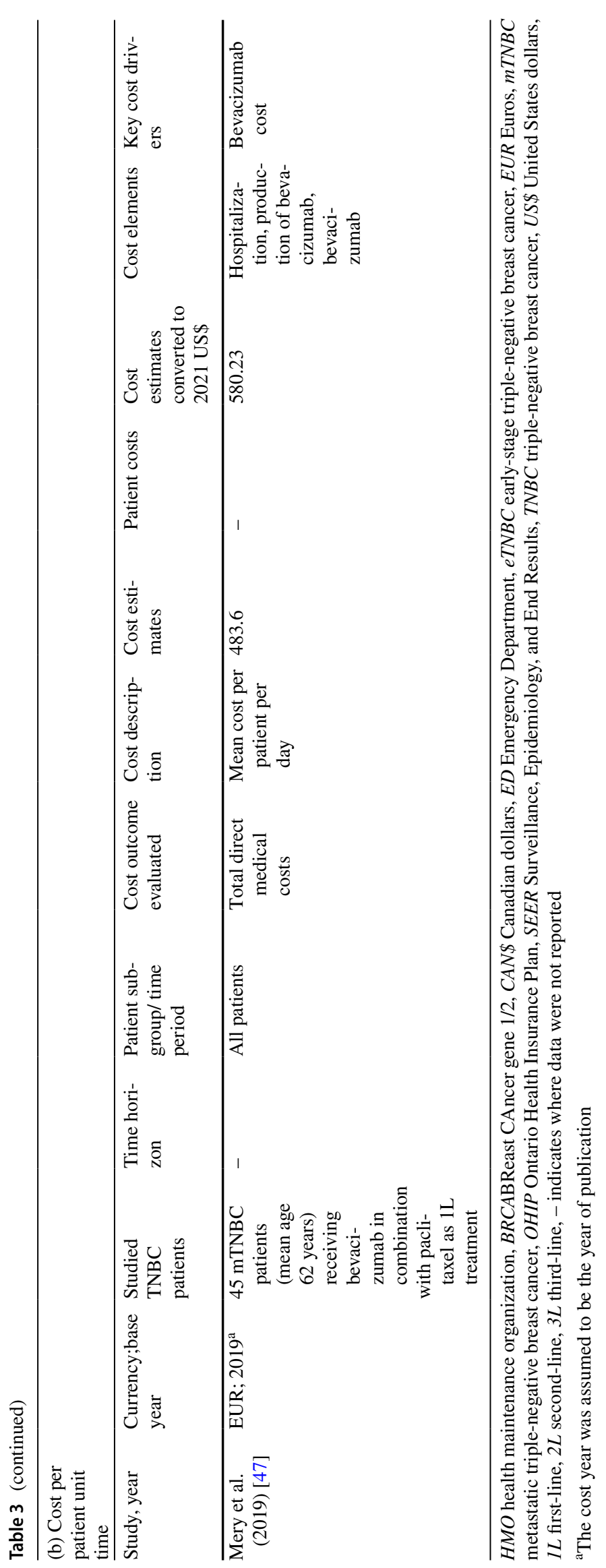




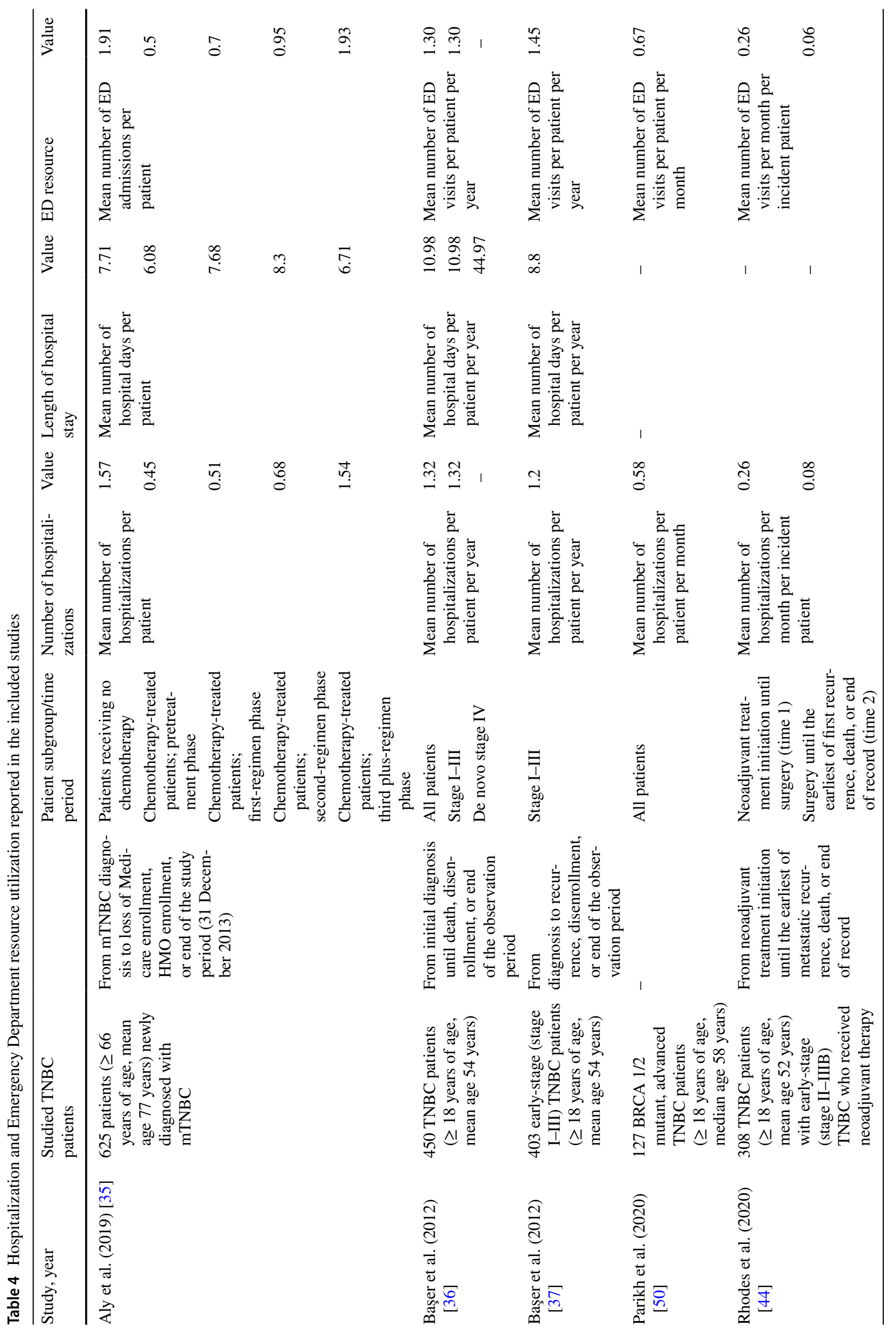




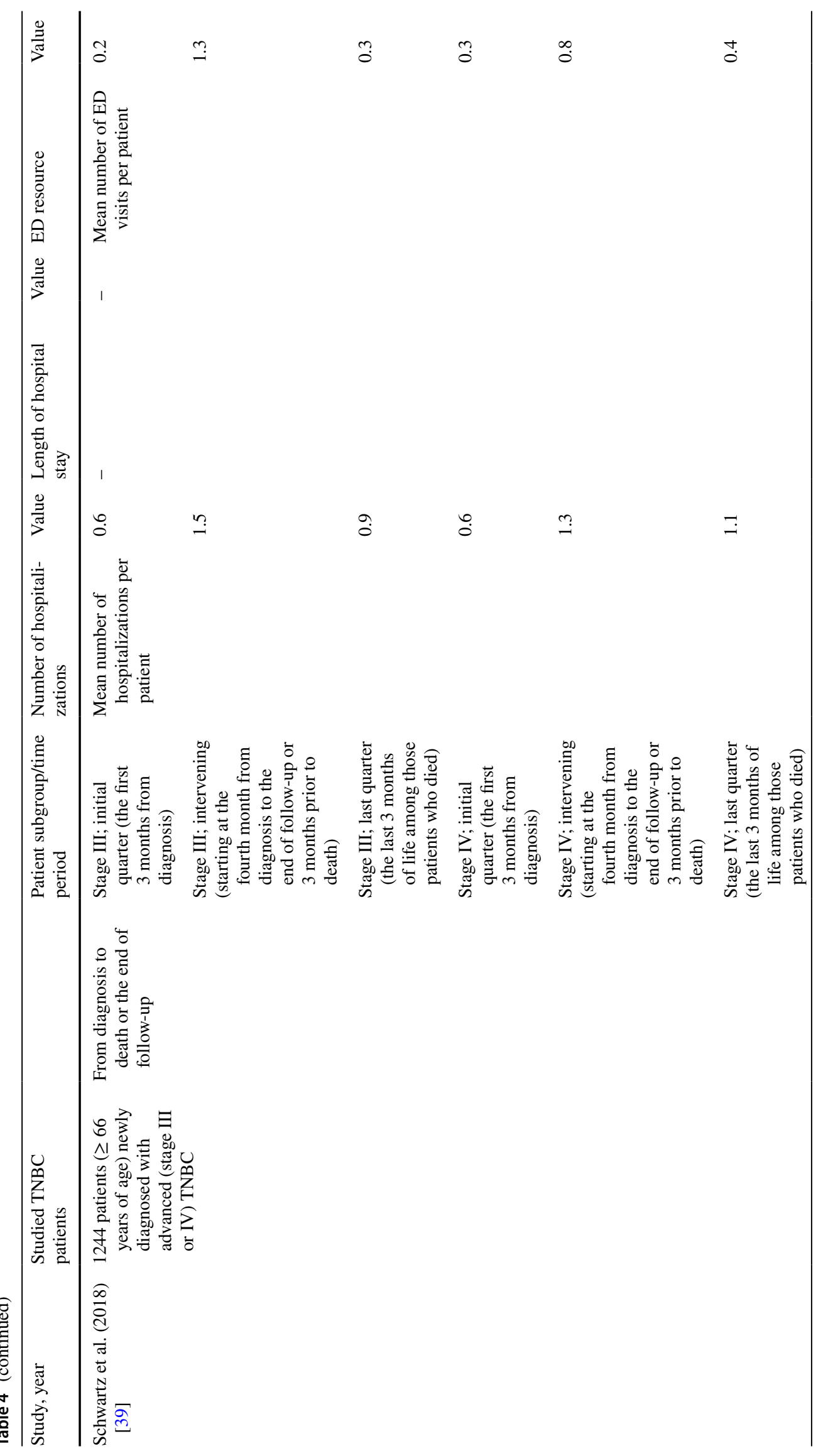




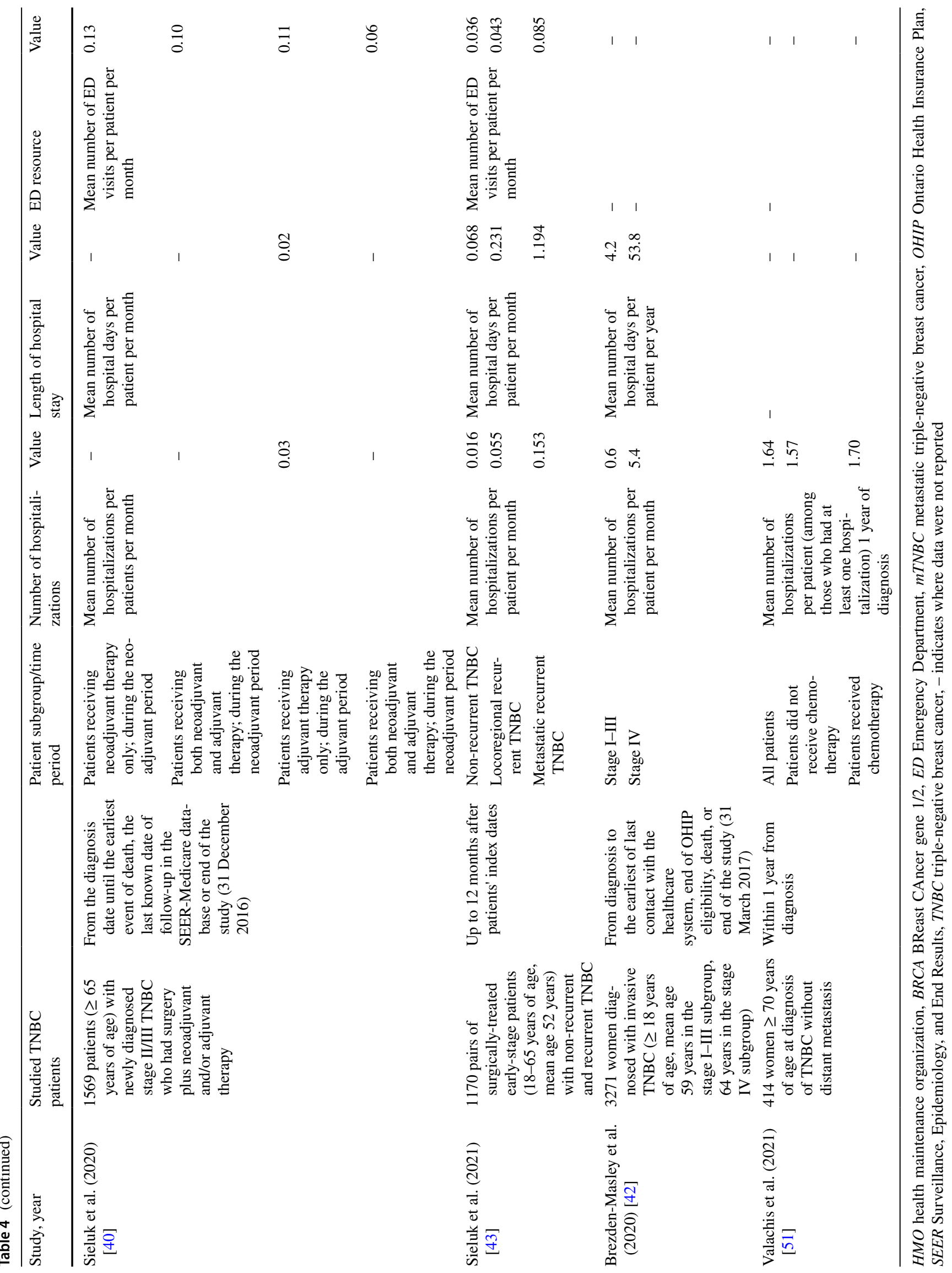


design, time horizon, patient population, treatment pattern, and healthcare systems.

On a per-unit time basis, estimates of the average perpatient annual direct medical cost ranged from about $\$ 20,000$ to over $\$ 100,000$ in stage I-III and around $\$ 100,000-\$ 300,000$ in stage IV TNBC, from the US healthcare payer perspective. Brezden-Masley et al. [42] estimated Canadian public health system-related costs in stage I-III and stage IV TNBC and the annual per-patient costs reported were $\$ 29,625$ and $\$ 118,418$, respectively. Based on a study in a single-center setting in France by Mery et al. [47], the average cost of treating an mTNBC patient who received first-line treatment with bevacizumab plus paclitaxel was $\$ 580$ per day. The highest estimate of over $\$ 300,000$ annual cost was based on the study by Skinner et al. [49] for patients during the third and later line of the treatment period of mTNBC, and the lowest estimate of about $\$ 20,000$ was based on the study by Rhodes et al. [44] in eTNBC after definitive surgery until the first recurrence. All costs reported above were adjusted to 2021 US\$.

The economic burden associated with cancer recurrence and progression was significant and greater costs were associated with increasing disease severity. A retrospective cohort study conducted by Sieluk et al. [43] evaluated HCRU and direct medical costs associated with recurrence in surgically treated eTNBC using the OptumHealth Reporting and Insights database. The adjusted cost models estimated that metastatic recurrent patients incurred $\$ 8322$ more in total monthly all-cause costs than non-recurrence patients, and locoregional recurrent patients incurred $\$ 3537$ (in 2019 US\$) more than non-recurrence patients $(p<0.001$ for both comparisons). Adjusted incidence rate ratios of all-cause inpatient admissions were 3.67 and 10.19 times higher for locoregional and metastatic cohorts, respectively ( $p<0.001$ for both comparisons). The locoregional recurrence cohort had a significantly higher rate of breast cancer surgery and the metastatic recurrence cohort had significantly higher chemotherapy use compared with the non-recurrence cohort. Brandão et al. [46] estimated direct medical costs for eTNBC care in the first 3 years after diagnosis in a Portuguese cancer center setting and reported that the median total cost per patient increased from stage I to stage III (€9566-€14,645 in 2015 Euros). Brezden-Masley et al. [42] conducted a retrospective study using a publicly funded healthcare system in Ontario to descriptively compare HCRU and healthcare costs by cancer stage (I-III vs. IV) in adult women diagnosed with invasive TNBC. The study underscored substantially higher costs associated with mTNBC, even considering the generally shorter treatment duration and a lack of more targeted and expensive biological treatments in this setting (total annual cost CAN\$35,064 in stage I-III versus CAN\$140,160 in stage IV; cost in 2017 CAN\$). Patients with mTNBC had more inpatient hospital visits and a substantially longer average length of stay (65.1 vs. 7.5 days) than eTNBC patients. Similar cost patterns were observed in other studies. [36, 37, 39]

The cost impact of mTNBC was substantial and increased with further lines of therapy and a shorter time to death. Aly et al. [35] identified patients diagnosed with mTNBC from the Surveillance, Epidemiology, and End Results (SEER)Medicare database. Within the chemotherapy-treated cohort, total direct medical costs per patient increased as the line of therapy increased (pretreatment: $\$ 13,188$; during the first regimen: $\$ 26,950$; during the second regimen: $\$ 33,347$; during the third or later regimen: $\$ 50,627$, in 2017 US\$), and greater ED and hospital utilization was observed in the third or later regimen phase. Skinner et al. [49] examined healthcare costs of mTNBC at community oncology practices and suggested that the estimates of per patient per month costs increased with further lines of therapy $(\$ 21,908, \$ 24,891$, and $\$ 25,845$ in 2017 US\$ during the first, second, and third or later lines, respectively). Sieluk et al. [41] highlighted poor outcomes and high costs associated with mTNBC using 2010-2016 SEER-Medicare data. Direct costs were categorized by the length of time to death. Total costs, as well as inpatient, ED, and hospice costs, substantially increased as the time period approached death.

Studies showed that patients who did not receive anticancer treatment had more comorbidities and shorter life expectancy. In addition, the mean monthly direct cost per patient was higher in patients with mTNBC who did not receive chemotherapy compared with those who did. [35] For patients who received cancer treatment, costs were generally high during initial treatment following diagnosis and decreased substantially during continuing care, but increased again in the terminal care stage, mainly for hospitalization. [39]

Most studies reported that the majority of healthcare costs were due to outpatient visits for early-stage patients, while ED and hospitalization were often the main cost drivers in the metastatic setting, especially in patients without anticancer treatments or towards the end of life. Several studies reported costs of anticancer therapies separately from the costs of cancer management. Mery et al. [47] reported that bevacizumab treatment costs comprised nearly $75 \%$ of total costs for the mTNBC patients who received bevacizumab plus paclitaxel as first-line treatment in a single center in France. Houts et al. [38] found systemic anticancer therapy accounted for approximately $50 \%$ of the total cost of care for $B R C A$-mutated mTNBC during first-line treatment in US community oncology settings, while hospitalization was the second largest cost driver. In contrast, studies that adopted longer time horizons suggested that cancer treatment accounted for only a small portion of the overall cost of cancer care. A cost-of-illness study by De las Heras et al. [45] evaluated the economic burden of mTNBC in Spain 
over 5 years following metastatic disease diagnosis. It found that over half of the total direct costs were attributable to palliative/best supportive care, while $<30 \%$ was attributable to active cancer treatment. Similarly, two studies conducted in the US [41, 49] suggested that cancer management for mTNBC was costly and systemic therapies accounted for only a small portion $(5-30 \%)$ of the total medical costs over patients' lifetime after diagnosis of metastatic disease. Rhodes et al. [44] examined the HCRU and costs in patients diagnosed with eTNBC (stage II-IIIB) at a US community oncology practice, which found that infused supportive care and systemic anticancer therapy were the primary cost drivers during the neoadjuvant treatment period; however, the majority of costs were attributable to hospitalization and ED visits during the remainder of the follow-up after surgery. Additionally, in the study by Brandão et al. [46], which followed eTNBC patients over 3 years after diagnosis in a Portuguese cancer center setting, only $7.5 \%$ of the cancer care cost was attributed to systemic therapy, while surgery, radiotherapy, and hospitalization accounted for the largest proportions of the total cost.

Patient Out-of-Pocket Costs The high cost of care for TNBC is not only a tremendous economic burden on healthcare systems but it also poses a substantial burden on patients.

Four studies in the review reported patient out-of-pocket expenditures in the US. The SEER-Medicare studies by Sieluk et al. [40, 41, 43] reported patient out-of-pocket expenses for anticancer therapy and inpatient, ED, outpatient, skilled nurse facility, and hospice visits in the Medicare setting. Average patient costs were over $\$ 1000$ per month in mTNBC and over $\$ 2000$ per month for eTNBC patients in both the neoadjuvant therapy and adjuvant therapy period. In the two studies by Baser et al. [36, 37], it was reported that health plans covered $75.5 \%$ of the total direct medical costs for patients with (stage I-III) eTNBC and $96.8 \%$ for (stage IV) mTNBC in a US national managed-care health plan setting. This led to annual patient costs of around $\$ 23,000$ and $\$ 5000$ in early-stage breast cancer and mTNBC, respectively.

\subsubsection{Indirect Costs}

The indirect costs of treating TNBC were assessed in only three studies. Two studies conducted in the US were conference presentations $[52,53]$ and one study conducted in Spain was a published manuscript [45]. An overview of indirect costs is shown in Table 5. While these studies demonstrated indirect costs related to productivity loss, the costs related to premature mortality and caregiver costs were not included.

Sieluk et al. [53] used the OptumHealth Reporting and Insights database to evaluate work loss and indirect costs due to medically-related absenteeism (ABS) and disability in early-stage surgically-treated TNBC patients with and without recurrence. Compared with the non-recurrence cohort, total work loss days were 1.51 and 2.08 times higher with locoregional and metastatic recurrence, respectively ( $p<0.001$ for both comparisons). Similarly, monthly productivity costs were significantly greater for patients with locoregional recurrence (\$849) and metastatic recurrence (\$1454) than patients without recurrence (\$451). Driven mainly by ABS and short- and long-term disability (STD and LTD, respectively), the rate of leaving the workforce was $63 \%$ greater in the recurrence cohort $(p=0.003)$.

Tabah et al. [52] assessed the indirect productivity burden of mTNBC using employed patients' ABS, STD, and LTD data at 6 months after the initiation of treatment. Among 56 patients with data eligibility, four patients had an ABS claim and missed a mean of 245 work hours, contributing to a mean productivity loss of $\$ 6472$ (in 2017 US\$) per patient. STD and LTD claims were identified in 14 and 4 patients, respectively, who missed a mean of 63 and 76 workdays, leading to a mean productivity loss of $\$ 9265$ and $\$ 11,192$, respectively.

The Spanish cost-of-illness study conducted by De las Heras et al. [45] also reported indirect costs measured by lost productivity due to missed workdays for the working population. Total mean indirect costs for patients with mTNBC were estimated to be $€ 164$ (in 2016 Euros) over 5 years following mTNBC diagnosis.

\subsection{The Humanistic Burden of TNBC}

Compared with the economic burden of TNBC, the humanistic burden is more difficult to quantify; still, it is critical in medical decisions, including managing patients' conditions, planning treatment pathways, and assessing the benefit-risk of treatment options.

Table $2 \mathrm{~b}$ displays the characteristics of the 10 studies published from 2012 to 2020 that assessed quality of life of TNBC patients, comprising five RCTs [54-58], two non-randomized clinical trials $[59,60]$, two cross-sectional studies [61-63], and one retrospective study [64]. Five of the studies assessed HRQoL outcome data using FACT-B, four studies reported EORTC QLQ-C30 and QLQ-BR23, and one study reported EQ-5D. Three studies were conducted in the US, one each in Italy and China, and the remaining studies were multinational. Four studies were published manuscripts, four were conference presentations, and two were both published manuscripts and presented at conferences. Most of the studies evaluated interventions for $\mathrm{mTNBC}$, where the objective of the interventions was not curative and the patient's wellbeing was essential. 


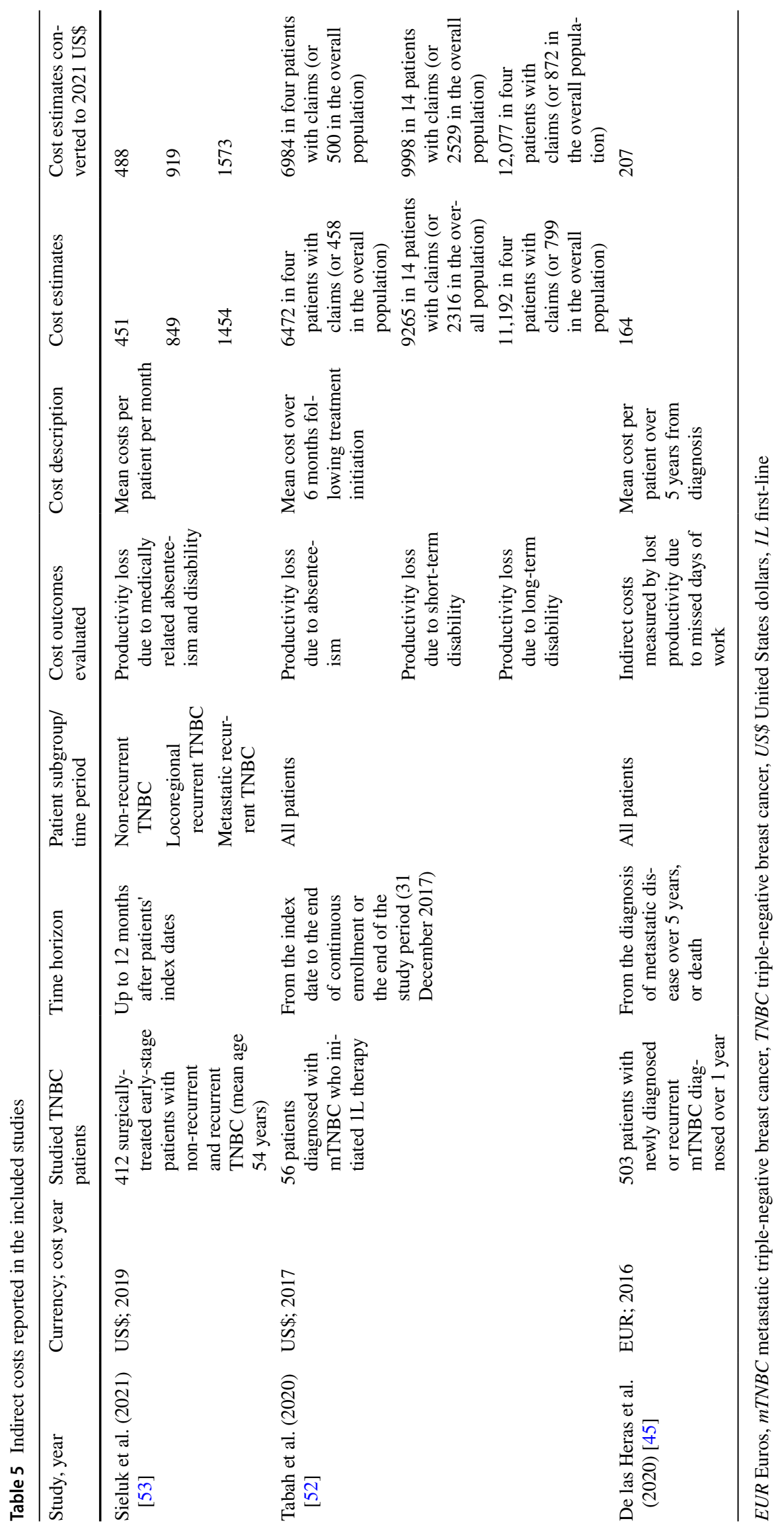




\subsubsection{Study Quality Assessment}

The five RCTs [54-58] had a low risk of bias in most domains in the Cochrane Risk of Bias Tool, except that risk of performance and detection biases was high in four studies due to the open-label trial design. The non-randomized trials $[59,60]$ had moderate quality with high risk in the NOS selection domain related to representativeness of the sample at the time points when HRQoL was evaluated. The cross-sectional studies [61-63] were rated as moderate quality with the adapted NOS, where the main risk of bias was lack of description of the derivation or representativeness of the included sample or the sample size calculation. Quality assessment for Mocerino et al. [64] was not undertaken as its English abstract and data tables did not contain sufficient information for assessment, while the remainder of the article was not in English.

\subsubsection{Health-Related Quality-of-Life Outcomes}

Treatment options affect patients' quality of life in various ways. An overview of HRQoL outcomes is presented in Table 6. While the evidence base was sparse, the RCTs showed that treatment with pembrolizumab and talazoparib statistically significantly improved $\mathrm{HRQoL}$ relative to chemotherapy on several instruments in PD-L1-positive mTNBC and BRCA-mutated TNBC, respectively. Exercise and dietary counseling programs were also reported to significantly improve HRQoL in TNBC survivors. In addition, levels of hope, self-efficacy, and social support were positive predictors of HRQoL.

EORTC QLQ-C30 and QLQ-BR23 EORTC QLQ-C30 is an instrument that assesses HRQoL in cancer patients using one global health scale, five functional scales (physical, role, emotional, social, cognitive), and eight symptom scales and single items (fatigue, pain, nausea/vomiting, appetite loss, constipation, diarrhea, insomnia, dyspnea) [65]. The EORTC QLQ-BR23 instrument is specific for BC patients and is comprised of 23 items to assess four functional scales (body image, sexual functioning, sexual enjoyment, future perspective) and four symptom scales (systemic therapy adverse effects, breast symptoms, arm symptoms, upset by hair loss) [66]. For both instruments, higher scores on functional scales suggest better status, while higher scores on symptom scales indicate worse symptoms. A difference of 10 points is suggested as the minimal clinically important difference for EORTC QLQ-C30 and QLQ-BR23 in BC patients. [67]

Three RCTs assessed HRQoL improvements using EORTC QLQ-C30 and QLQ-BR23. The phase III IMpassion130 trial compared the combination of atezolizumab and nab-paclitaxel with placebo and nab-paclitaxel as firstline treatment in patients with mTNBC [54]. No significant differences between arms in time to deterioration (TTD) were observed for physical or role functioning or global health scale in PD-L1-positive patients. Change from baseline values for treatment symptom scales was also comparable between arms. The phase III KEYNOTE-119 trial compared pembrolizumab with investigator's choice of chemotherapy in previously treated mTNBC patients [57]. In the PD-L1-positive (combined positive score [CPS] $\geq 10$ ) subgroup, there were statistically significant differences between treatment arms in change from baseline values for the global health scale, physical functioning scale, and nausea/vomiting and diarrhea in symptom scales from the QLQ$\mathrm{C} 30$ instrument, and the systemic therapy adverse effects scale on the QLQ-BR23 instrument measured at 6 weeks from baseline. From the phase III EMBRACA trial, which compared talazoparib with physician's choice of chemotherapy in patients with advanced TNBC and a germline $B R C A$ mutation [56], a statistically significant difference in change from baseline in global health status/quality of life and several functioning and symptom scales on the QLQC30 was observed between treatment arms.

$F A C T-B$ FACT-B is an instrument that assesses five domains of HRQoL in BC patients (physical well-being, social well-being, emotional well-being, functional wellbeing, and BC subscale) [68]. Negative items are reverse scored such that higher scores suggest greater health. The minimal clinically important difference for FACT-B is estimated to be 7-8 points. [69]

The studies by Vadaparampil et al. [62, 63] showed significantly lower FACT-B total scores in TNBC participants compared with non-TNBC participants and demonstrated clinically meaningful differences in HRQOL among TNBC and non-TNBC patients. Significant associations were also reported between FACT-B scores and income, chemotherapy, current health, role limitation, anxiety, life stress, collectivism, and fatalism. Shen et al. [61] showed positive correlations between HRQoL and hope, social support, and self-efficacy.

Swisher et al. [58] reported that supervised moderateintensity aerobic exercise and diet counseling in an RCT led to significant HRQoL improvements from baseline in TNBC survivors regarding physical, emotional well-being, breast cancer subscale, and FACT-B total scores; however, no significant improvement in FACT-B scores was observed for TNBC interventions investigated in other studies. [59, $60,64,70]$

\subsubsection{Health State Utility Values}

There is a paucity of health state utility values associated with TNBC. One study by Huang et al. [55] reported utility values in previously treated mTNBC patients derived from EQ-5D-3L data collected in the phase III KEYNOTE-119 


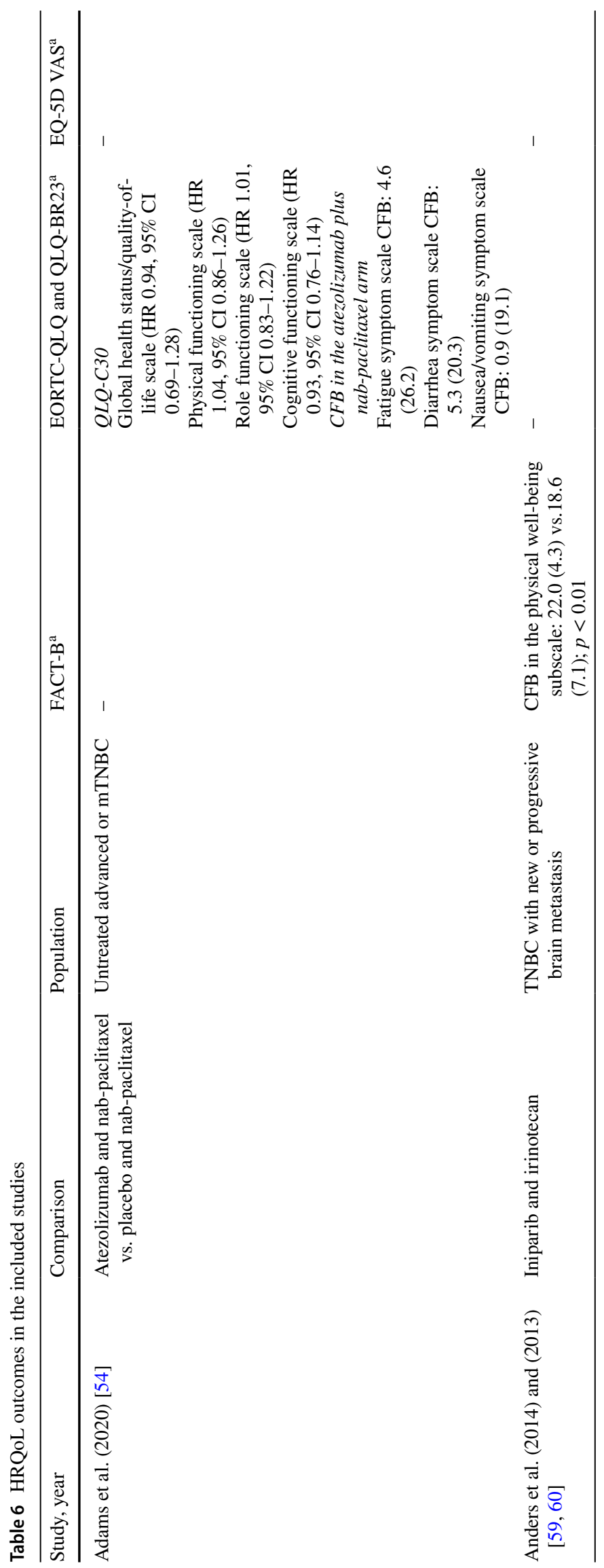




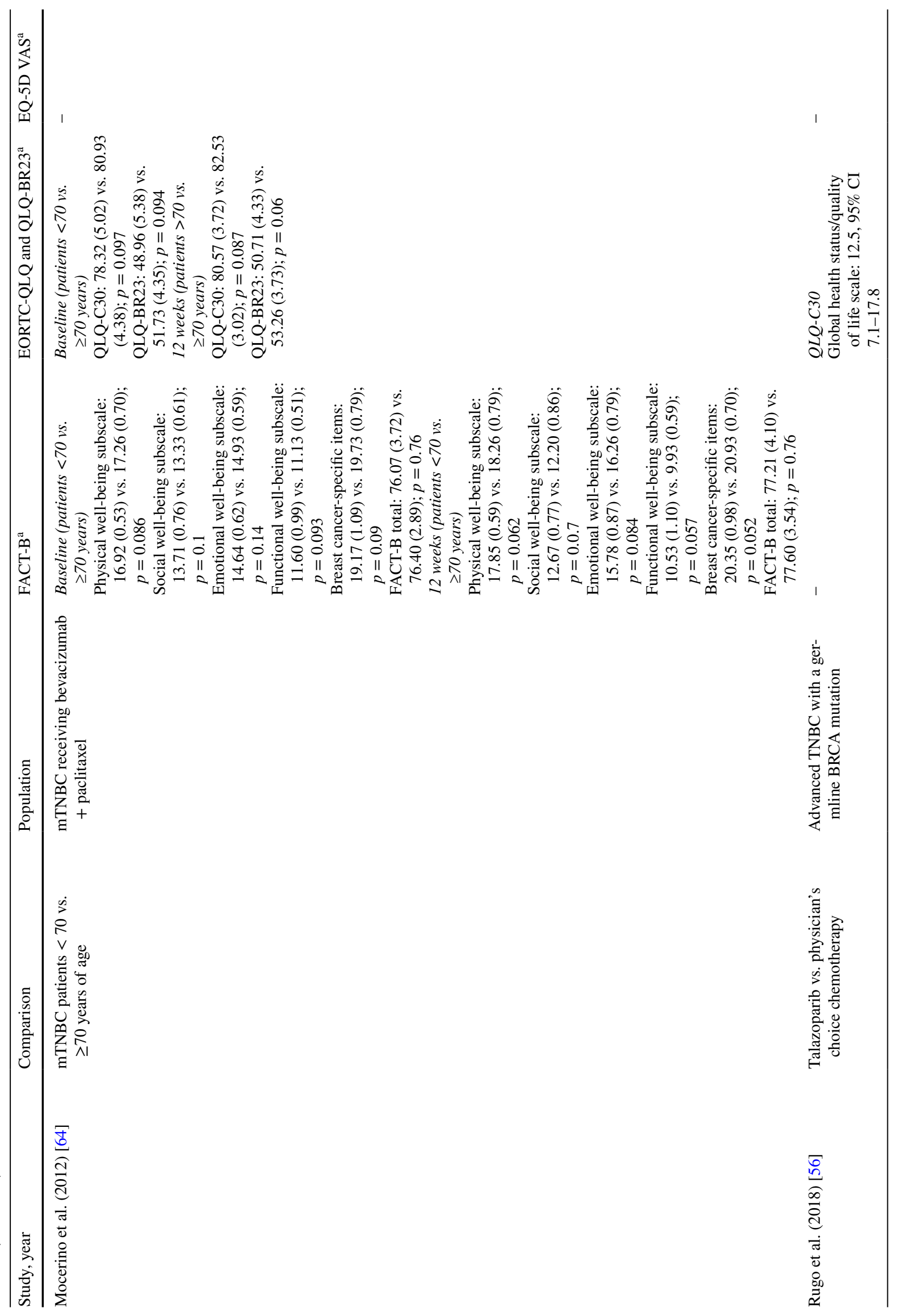




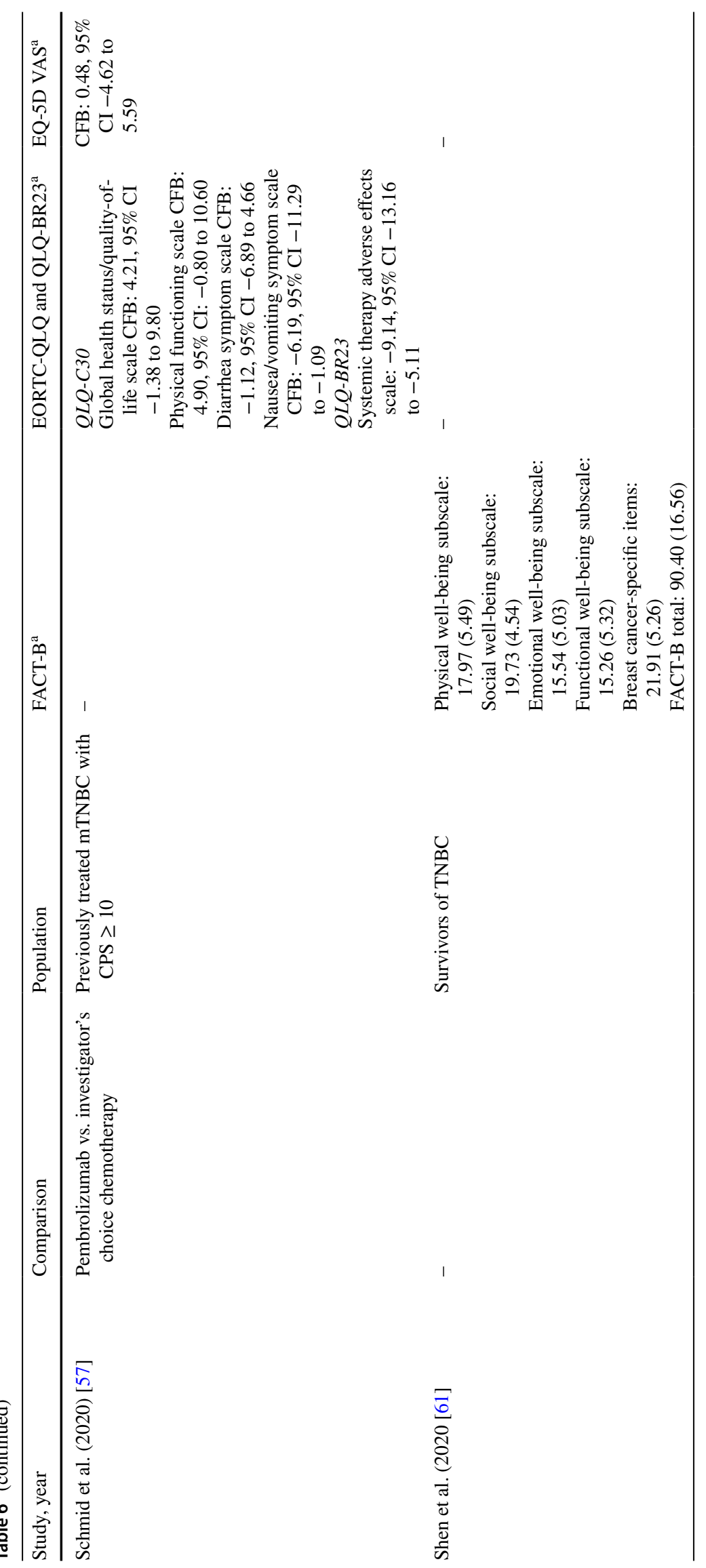




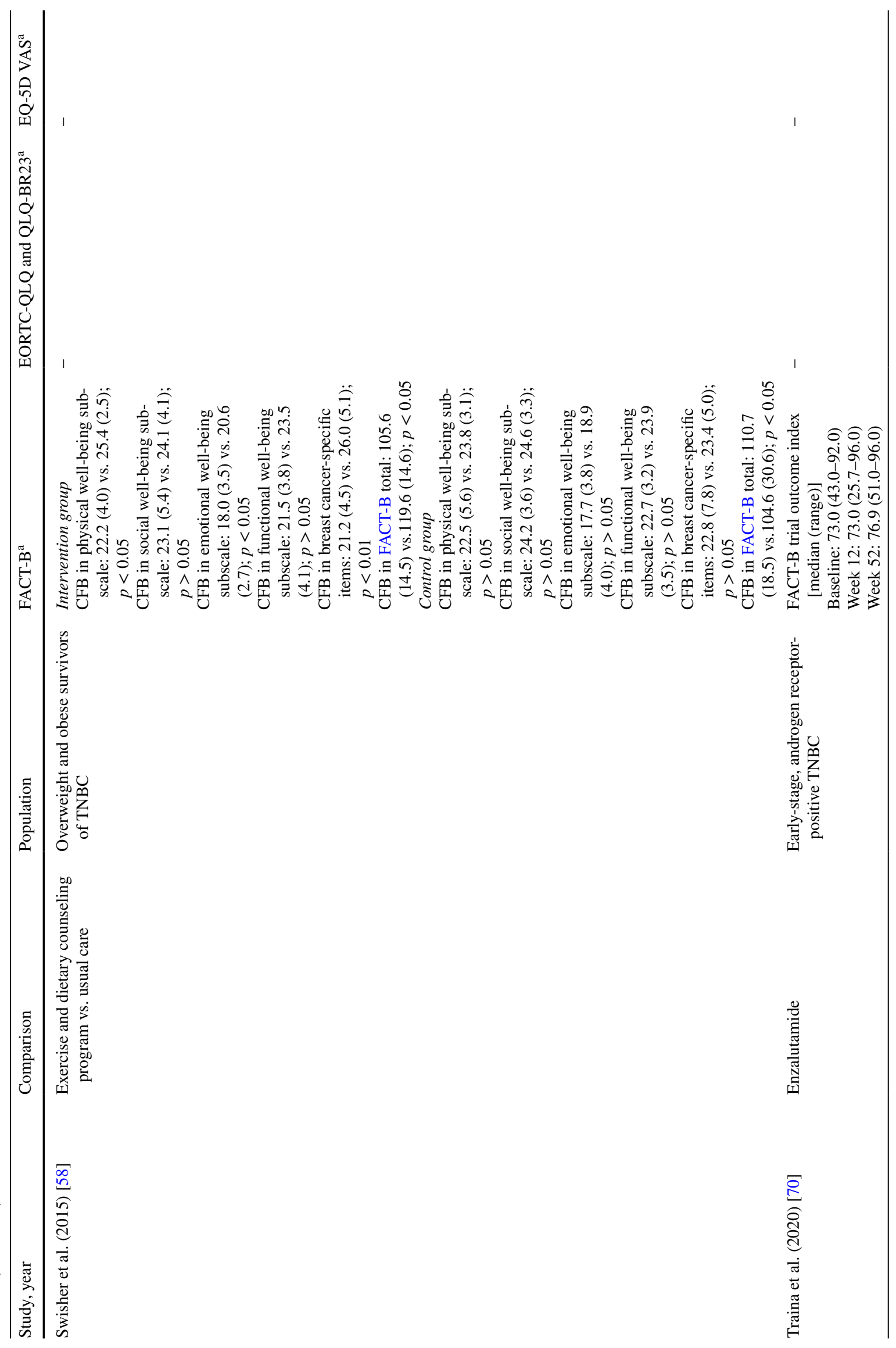




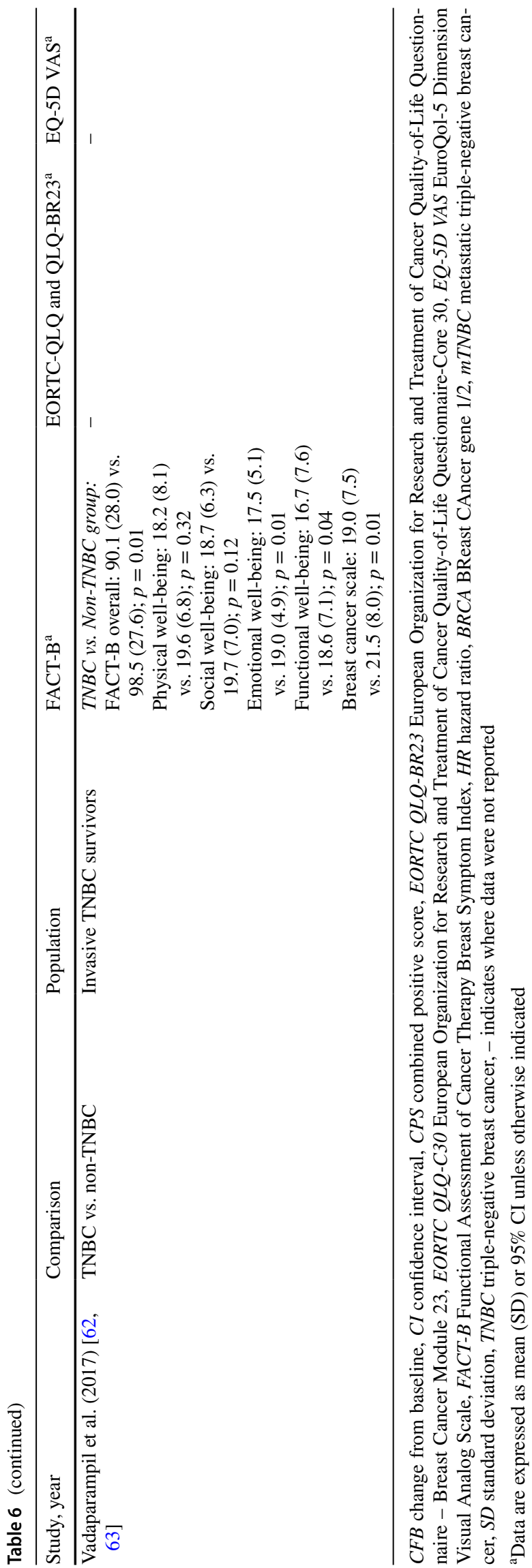

trial (see Table 7). Mean utility for patients in progressionfree and progressive disease states was 0.715 (95\% CI $0.701-0.730)$ and 0.601 (95\% CI 0.571-0.631), respectively. In this study, utility values were also estimated by time-todeath category. The results showed a rapid deterioration of quality of life in mTNBC patients towards the end of life. The utility decreases associated with disease progression and time-to-death were clinically meaningful.

\section{Discussion}

To our knowledge, this is the largest and most comprehensive SLR that synthesized the literature on the economic and humanistic burden of TNBC. While one targeted literature review (Naidoo et al. [71]) and four SLRs (Frederickson et al. [73], Frederickson et al. [74], Fu et al. [72], and Parisi et al. [29]) were identified in our search, the evidence base with particular focus on TNBC was limited in these studies. With the rapid growth of research in this area, our SLR was based on a broader set of evidence and included a total of 19 unique studies assessing the costs and HCRU and 10 studies assessing HRQoL in TNBC patients.

HCRU and the direct medical costs associated with TNBC were substantial and increased with disease severity. For patients diagnosed with eTNBC, monthly costs of cancer care were estimated to be three and seven times greater after locoregional and metastatic recurrence, respectively. [43]

Considerable variations in cost estimates were observed across studies. Estimates of the average per-patient annual direct medical cost ranged from about $\$ 20,000$ to over $\$ 100,000$ in stage I-III and around $\$ 100,000-\$ 300,000$ in stage IV TNBC. The US and Canadian studies appeared to report higher costs than those conducted in European countries. This could be due to differences in treatment guidelines and the nature of the healthcare systems, as well as the patient populations evaluated in the studies. Within the US studies, four assessed patients aged 65 years and older from Medicare claims data [40, 41, 43, 53], which reported relatively lower costs compared with other studies that included younger patients with commercial health plans. Cost estimates across the studies are not generally comparable as they are highly heterogeneous in study setting, patient populations, time horizons, and cost elements evaluated.

Many studies found that outpatient visits accounted for a majority of the direct medical costs for early-stage patients. In contrast, $\mathrm{ED}$ visits and hospitalization were the main cost drivers in the metastatic setting, especially in patients without anticancer treatments or towards the end of life. Regarding anticancer therapy costs, four US studies and three ex-US studies reported such costs separately from the costs of cancer care management. Most of these studies concluded that the majority of the total medical costs were 
Table 7 Health state utility values reported in the included studies

\begin{tabular}{|c|c|c|c|c|c|}
\hline Study, year & Population & Progression category & $\begin{array}{l}\text { Mean }(95 \% \text { CI) utility } \\
\text { value }\end{array}$ & $\begin{array}{l}\text { Time-to-death } \\
\text { category, days }\end{array}$ & $\begin{array}{l}\text { Mean }(95 \% \text { CI }) \text { utility } \\
\text { value }\end{array}$ \\
\hline \multirow[t]{5}{*}{ Huang et al. (2020) [55] } & \multirow[t]{5}{*}{$\begin{array}{l}\text { Patients with previously } \\
\text { treated mTNBC }\end{array}$} & \multirow[t]{2}{*}{$\begin{array}{l}\text { Progression-free } \\
\text { survival }\end{array}$} & \multirow[t]{2}{*}{$\begin{array}{l}0.715(95 \% \text { CI } 0.701- \\
\quad 0.730)\end{array}$} & $>360$ & $\begin{array}{l}0.765(95 \% \mathrm{CI} \\
0.750-0.779)\end{array}$ \\
\hline & & & & $180-360$ & $\begin{array}{l}0.655(95 \% \mathrm{CI} \\
0.624-0.687)\end{array}$ \\
\hline & & \multirow[t]{3}{*}{ Progressive disease } & \multirow[t]{3}{*}{$\begin{array}{l}0.601(95 \% \text { CI } 0.571- \\
\quad 0.631)\end{array}$} & $90-180$ & $\begin{array}{l}0.586(95 \% \text { CI } \\
0.549-0.624)\end{array}$ \\
\hline & & & & $30-90$ & $\begin{array}{l}0.517(95 \% \mathrm{CI} \\
0.471-0.564)\end{array}$ \\
\hline & & & & $>30$ & $\begin{array}{l}0.264(95 \% \text { CI } \\
0.128-0.401)\end{array}$ \\
\hline
\end{tabular}

$C I$ confidence interval, $m T N B C$ metastatic triple-negative breast cancer

from cancer management and that only about 5-30\% was due to systemic anticancer therapy. The findings reflected the clinical reality when traditional chemotherapies were the primary systemic therapies for TNBC. With the emergence of new cancer therapies, such as immune-oncology treatments for TNBC, the cancer treatment costs will undoubtably increase. On the other hand, the emerging therapies may reduce HCRU related to cancer management, including ED and hospitalization visits, due to better disease control. In addition, these therapies will significantly reduce cancer recurrence and progression, thereby decreasing annual medical costs per patient. Further research is desirable to evaluate the cost impact and cost effectiveness of the novel therapies, especially immunotherapies, in the real-life management of TNBC.

Most studies focused on costs paid by healthcare payers. Four studies reported patient out-of-pocket costs, ranging from approximately $\$ 500$ (in mTNBC) to $\$ 2000$ (in eTNBC) per month in the US [36, 37, 40, 41, 43]. The reported costs did not take into account other expenditures such as needed informal care or caregiver costs. These out-of-pocket costs added a considerable burden to the TNBC patients who were already suffering from decreased work productivity, reduced income, impaired quality of life, and increased disability.

Although indirect costs were sparsely reported, studies reporting cancer-related workplace absenteeism and disability suggested these were also important cost components from patient and societal perspectives. For example, the study by Sieluk et al. [53] reported monthly productivity costs ranging from around $\$ 500$ to $\$ 1500$. Disease recurrence was associated with decreased productivity and higher rates of leaving the workforce. Furthermore, the incidence of TNBC is high among women under the age of 65 years, which may substantially increase the risk of productivity loss and lead to significant indirect costs due to disability and early retirement among working-age women. While these studies demonstrated indirect costs related to productivity loss, costs related to premature mortality, comorbidity, and caregiver costs were not included. Thus, the overall indirect costs and societal burden of TNBC were likely underestimated.

Studies evaluating the humanistic burden of TNBC were primarily randomized or non-randomized clinical trials conducted internationally or in the US. Among the RCTs investigating HRQoL in mTNBC patients using EORTC QLQC30, QLQ-BR23, and FACT-B questionnaires, treatment with pembrolizumab and talazoparib showed significantly greater improvements in HRQoL compared with chemotherapy in PD-L1-positive mTNBC and BRCA-mutated mTNBC, respectively. [54, 56, 57] Another RCT found that a diet and exercise program among TNBC survivors also led to HRQoL benefits [58]. Health utility values specific to TNBC were only reported in one study in the literature, which included previously treated (progressed after first line) mTNBC patients in an RCT [44]. While an SLR presented at a conference identified health utility values used in cost-effectiveness analyses of TNBC interventions, the authors of those studies elected to use utility estimates from general BC patients [73]. Mean utility values for pretreated mTNBC were reported much lower in the SLR compared with the RCT (mean 0.45 vs. 0.715 ), which could be due to differences in the patient populations. Furthermore, the utility values cited in the SLR were based on an older study conducted in Sweden in 2005, which did not reflect the development of new, less toxic, and more efficacious therapeutic options for BC during the past 15 years since the study was conducted. Despite the difference, both studies showed a rapid deterioration in quality of life in $\mathrm{mTNBC}$ patients, and a significant decrease in health utility associated with disease progression.

This study has several limitations. First, we adjusted reported costs to 2021 US\$ to facilitate comparisons across studies; however, this conversion can be imperfect when practice patterns and inflation rates differ between countries. 
Second, all SLRs are limited by publication bias with respect to the available articles. Third, with the development of novel therapies to treat patients with TNBC, older economic evaluations and cost estimates may become dated, highlighting the need for regular evidence synthesis of the available literature to facilitate understanding of the burden of TNBC. Lastly, the review includes studies from different countries and regions. Thus, the studies are heterogeneous and the results are not easily generalizable.

\section{Conclusion}

TNBC poses a tremendous economic burden on healthcare systems and societies globally, with substantially greater costs associated with increasing disease severity. TNBC patients experience reduced HRQoL, decreased productivity, and rising out-of-pocket expenses.

ED visits and hospitalization were commonly reported as the main cost drivers in mTNBC studies, suggesting that focusing on hospital and terminal care could be imperative for managing overall costs in the late stage of the disease. TNBC care is changing rapidly, with immunotherapies emerging as a new treatment paradigm. The new therapies impose higher treatment costs but will reduce the need for subsequent therapies and other healthcare resources because of better disease control, improved HRQoL, and delayed cancer recurrence. Additional research is required to evaluate the impact of these therapies on the economic and humanistic burden of TNBC to assist medical decisions for healthcare payers, providers, and patients.

Supplementary Information The online version contains supplementary material available at https://doi.org/10.1007/s40273-021-01121-7.

\section{Declarations}

Funding This study was funded by Merck \& Co., Inc.

Conflicts of interest Min Huang and Amin Haiderali are employees of Merck \& Co. Inc., the sponsor of this study and corresponding manuscript. Grace E. Fox and Andrew Frederickson are employees of PRECISIONheor, a healthcare research consultancy that has received funding from Merck and Co., Inc. Javier Cortes provides advisory and consultancy services to Roche, Celgene, Cellestia, AstraZeneca, Seattle Genetics, Daiichi Sankyo, Erytech, Athenex, Polyphor, Lilly, Merck Sharp \& Dohme, GSK, Leuko, Bioasis, Clovis Oncology, Boehringer Ingelheim, Ellipses, Hibercell, BioInvent, Gemoab, and Gilead. Peter A. Fasching reports personal fees from Novartis Pfizer, Daiichi-Sankyo, AstraZeneca, Eisai, Merck Sharp \& Dohme, Lilly Pierre Fabre, SeaGen, Roche, Hexal, and Agendia, and grants from Biontech and Cepheid. Joyce O'Shaughnessy receives consulting fees and/or honorarium from AbbVie Inc., Agendia, Amgen Biotechnology, Aptitude Health, AstraZeneca, Bayer, Bristol-Myers Squibb, Celgene Corporation, Clovis Oncology, Daiichi Sankyo, Eisai, G1 Therapeutics, Genentech, Gilead Sciences, GRAIL, Halozyme Therapeutics, Heron Therapeutics, Immunomedics, Ipsen Biopharmaceuticals, Lilly,
Merck, Myriad, Nektar Therapeutics, Novartis, Pfizer, Pharmacyclics, Pierre Fabre Pharmaceuticals, Puma Biotechnology, Prime Oncology, Roche, Samsung Bioepis, Sanofi, Seagen, Syndax Pharmaceuticals, Taiho Oncology, Takeda, and Synthon.

Ethical Approval Not applicable.

Consent for publication Not applicable.

Consent for participate Not applicable.

Availability of data and material All included publications were obtained through publicly available sources. All data generated or analyzed during this study are included in this published article or the electronic supplementary material.

Code availability Not applicable.

Author contributions All authors contributed to the study design and data analyses, interpretation of the results, and development and writing of the draft manuscript, and approved the final version.

Open Access This article is licensed under a Creative Commons Attribution-NonCommercial 4.0 International License, which permits any non-commercial use, sharing, adaptation, distribution and reproduction in any medium or format, as long as you give appropriate credit to the original author(s) and the source, provide a link to the Creative Commons licence, and indicate if changes were made. The images or other third party material in this article are included in the article's Creative Commons licence, unless indicated otherwise in a credit line to the material. If material is not included in the article's Creative Commons licence and your intended use is not permitted by statutory regulation or exceeds the permitted use, you will need to obtain permission directly from the copyright holder. To view a copy of this licence, visit $\mathrm{http} / / /$ creativecommons.org/licenses/by-nc/4.0/.

\section{References}

1. Sung H, Ferlay J, Siegel RL, et al. Global cancer statistics 2020: GLOBOCAN estimates of incidence and mortality worldwide for 36 cancers in 185 countries. CA Cancer J Clin. 2021;71(3):209-49.

2. Yersal O, Barutca S. Biological subtypes of breast cancer: Prognostic and therapeutic implications. World J Clin Oncol. 2014;5(3):412-24.

3. Waks AG, Winer EP. Breast Cancer Treatment: A Review. JAMA. 2019;321(3):288-300.

4. Foulkes WD, Smith IE, Reis-Filho JS. Triple-Negative Breast Cancer. N Engl J Med. 2010;363(20):1938-48.

5. Merck. FDA Approves Merck's KEYTRUDA ${ }^{\circledR}$ (pembrolizumab) in Combination With Chemotherapy for Patients With Locally Recurrent Unresectable or Metastatic Triple-Negative Breast Cancer Whose Tumors Express PD-L1 (CPS $\geq 10$ ). Merck. Available at: https://www.merck.com/news/fda-approves-mercks-keytruda-pembr olizumab-in-combination-with-chemotherapy-for-patients-with-local ly-recurrent-unresectable-or-metastatic-triple\%e2\%80\%91 negativebreast-cancer-whose/. Published 2020. Accessed 19 Nov 2021.

6. Merck. FDA Approves KEYTRUDA ${ }^{\circledR}$ (pembrolizumab) for Treatment of Patients With High-Risk Early-Stage Triple-Negative Breast Cancer in Combination With Chemotherapy as Neoadjuvant Treatment, Then Continued as Single Agent as Adjuvant Treatment After Surgery. Merck. Available at: https://www.merck.com/ news/fda-approves-keytruda-pembrolizumab-for-treatment-of-patie 
nts-with-high-risk-early-stage-triple-negative-breast-cancer-incombination-with-chemotherapy-as-neoadjuvant-treatment-thencontinued/. Published 2021. Accessed 19 Nov 2021.

7. Merck. European Commission Approves Merck's KEYTRUDA ${ }^{\circledR}$ (pembrolizumab) Plus Chemotherapy as Treatment for Certain Patients With Locally Recurrent Unresectable or Metastatic Triple-Negative Breast Cancer (TNBC). Merck. Available at: https:// www.merck.com/news/european-commission-approves-merckskeytruda-pembrolizumab-plus-chemotherapy-as-treatment-forcertain-patients-with-locally-recurrent-unresectable-or-metas tatic-triple-negative-breast/. Accessed 19 Nov 2021.

8. Clinical Practice Guidelines in Oncology (NCCN Guidelines). Breast Cancer. National Comprehensive Cancer Network. Available at: www.nccn.org/professionals/physician_gls/pdf/breast.pdf. Published 2021. Accessed 21 June 2021.

9. Cardoso F, Paluch-Shimon S, Senkus E, et al. 5th ESO-ESMO international consensus guidelines for advanced breast cancer (ABC 5). Ann Oncol. 2020;31(12):1623-49.

10. Cardoso F, Kyriakides S, Ohno S, et al. Early breast cancer: ESMO Clinical Practice Guidelines for diagnosis, treatment and follow-up. Ann Oncol. 2019;30(8):1194-220.

11. Montero AJ, Eapen S, Gorin B, Adler P. The economic burden of metastatic breast cancer: a U.S. managed care perspective. Breast Cancer Res Treat. 2012;134(2): 815-822.

12. Foster TS, Miller JD, Boye ME, Blieden MB, Gidwani R, Russell MW. The economic burden of metastatic breast cancer: a systematic review of literature from developed countries. Cancer Treat Rev. 2011;37(6):405-15.

13. Yabroff KR, Lund J, Kepka D, Mariotto A. Economic burden of cancer in the United States: estimates, projections, and future research. Cancer Epidemiol Biomark Prev. 2011;20(10):2006-14.

14. John R, Ross H. The global economic cost of cancer. American Cancer Society; 2010.

15. Radice D, Redaelli A. Breast Cancer Management. Pharmacoeconomics. 2003;21(6):383-96.

16. Dietze EC, Sistrunk C, Miranda-Carboni G, O'Regan R, Seewaldt VL. Triple-negative breast cancer in African-American women: disparities versus biology. Nat Rev Cancer. 2015;15(4):248-54.

17. Warren JL, Yabroff KR, Meekins A, Topor M, Lamont EB, Brown ML. Evaluation of trends in the cost of initial cancer treatment. J Natl Cancer Inst. 2008;100(12):888-97.

18. Zhuo J, Wu E, Dasbach EJ, Pellissier J. PCN190 Cancer Associated Medical Spending: US National Data Between 2000 and 2013. Value Health. 2016;19(7):A742-3.

19. Barron JJ, Quimbo R, Nikam PT, Amonkar MM. Assessing the economic burden of breast cancer in a US managed care population. Breast Cancer Res Treat. 2008;109(2):367-77.

20. Berkowitz N, Gupta S, Silberman G. Estimates of the lifetime direct costs of treatment for metastatic breast cancer. Value Health. 2000;3(1):23-30.

21. Iragorri N, de Oliveira C, Fitzgerald N, Essue B. The out-ofpocket cost burden of cancer care-a systematic literature review. Curr Oncol. 2021;28(2):1216-48.

22. Ekwueme DU, Trogdon JG, Khavjou OA, Guy GP Jr. Productivity costs associated with breast cancer among survivors aged 18-44 years. Am J Prev Med. 2016;50(2):286-94.

23. Holmstrom SHN, Dam S, Aballea S, Oestreicher N, Evans C, Novak A, Dydo M. Elicitation of health-related quality-of-life concepts associated with triple-negative breast cancer. Value in Health. 2015;18(3): 212.

24. Holmstrom S, Hawken NA, Dam S, et al. Elicitation of healthrelated quality-of-life concepts associated with triple-negative breast cancer. Value Health. 2015;18(3):A212.

25. Mokhatri-Hesari P, Montazeri A. Health-related quality of life in breast cancer patients: review of reviews from 2008 to 2018. Health Qual Life Outcomes. 2020;18(1):338.
26. Swiger K SJ, Guglielmino J. Emotional/psychological characteristics of women with triple-negative breast cancer: Do socioeconomic, demographic, and provider variables impact emotional change from diagnosis to post-treatment? Cancer Res. 75(9):615.

27. Vadaparampil ST, Christie J, Donovan KA, et al. Health-related quality of life in black breast cancer survivors with and without triple negative breast cancer (TNBC). Breast Cancer Res Treat. 2017;163(2):331-42.

28. Swiger KD, Sendecki JA, Guglielmino JE, et al. Abstract P5-1706: Emotional/psychological characteristics of women with triple-negative breast cancer: Do socioeconomic, demographic, and provider variables impact emotional change from diagnosis to post-treatment? Cancer Res. 2015;75(9 Suppl):P5-17-06.

29. Parisi M, Pelletier C, Cherepanov D, Broder MS. Outcomes research examining treatments, quality of life and costs in HER2negative and triple-negative metastatic breast cancer: a systematic literature review. J Comp Effect Res. 2018;7(1):67-83.

30. Moher D, Liberati A, Tetzlaff J, Altman DG. Preferred reporting items for systematic reviews and meta-analyses: the PRISMA statement. BMJ. 2009;339:b2535.

31. Higgins JPT, Altman DG, Gøtzsche PC, et al. The Cochrane Collaboration's tool for assessing risk of bias in randomised trials. BMJ. 2011;343:d5928.

32. Wells GS, O'Connell D, Peterson J, Welch V, Losos M. The Newcastle-Ottawa Scale (NOS) for assessing the quality of nonrandomised studies in meta-analyses. 2013. http://www.ohri.ca/progr ams/clinical_epidemiology/oxford.asp. Accessed 1 Oct 2016.

33. Turner HC, Lauer JA, Tran BX, Teerawattananon Y, Jit M. Adjusting for inflation and currency changes within health economic studies. Value Health. 2019;22(9):1026-32.

34. Medical consumer price index. US Bureau of Labor Statistics. Available at: https://www.bls.gov/cpi/factsheets/medical-care.htm. Accessed 5 Jul 2021.

35. Aly A, Shah R, Hill K, Botteman MF. Overall survival, costs and healthcare resource use by number of regimens received in elderly patients with newly diagnosed metastatic triple-negative breast cancer. Future Oncol. 2019;15(9):1007-20.

36. Başer O, Wei W, Henk HJ, Teitelbaum A, Xie L. Patient survival and healthcare utilization costs after diagnosis of triple-negative breast cancer in a United States managed care cancer registry. Curr Med Res Opin. 2012;28(3):419-28.

37. Başer O, Wei W, Henk HJ, Teitelbaum A, Xie L. Burden of earlystage triple-negative breast cancer in a US managed care plan. Health Outcomes Res Med. 2012;3(2):e57-65.

38. Houts AC, Olufade T, Shenolikar R, Walker MS, Schwartzberg LS. Treatment patterns, clinical outcomes, health resource utilization, and cost in patients with BRCA-mutated metastatic breast cancer treated in community oncology settings. Cancer Treat Res Commun. 2019;19:100121.

39. Schwartz KL, Simon MS, Bylsma LC, et al. Clinical and economic burden associated with stage III to IV triple-negative breast cancer: A SEER-Medicare historical cohort study in elderly women in the United States. Cancer. 2018;124(10):2104-14.

40. Sieluk J, Haiderali A, Huang M, Yang L, Hirshfield KM. Early triple-negative breast cancer in women aged $\geq 65$ : retrospective study of outcomes, resource use and costs, 2010-2016. Future Oncol. 2020;17(9):1039-54.

41. Sieluk J, Yang L, Haiderali A, Huang M, Hirshfield KM. Systemic therapy, survival and end-of-life costs for metastatic triple-negative breast cancer: retrospective SEER-Medicare study of women age $\geq 65$ years. Future Oncol. 2021;17(20):2581-92.

42. Brezden-Masley C, Fathers KE, Coombes ME, Pourmirza B, Xue C, Jerzak KJ. A population-based comparison of treatment patterns, resource utilization, and costs by cancer stage for Ontario patients with triple-negative breast cancer. Cancer Med. 2020;9(20):7548-57. 
43. Sieluk J, Haiderali A, Huang M, et al. PCN240 Healthcare Resource Utilization Associated With Disease Recurrence Among Surgically-Treated Patients With Triple-Negative Breast Cancer. Value in Health. 2021;24:S65.

44. Rhodes W, Gautam S, Haiderali A, et al. 104P Cost and healthcare resource utilization (HCRU) for patients receiving neoadjuvant therapy for early-stage triple-negative breast cancer (ESTNBC). Ann Oncol. 2020;31:S51.

45. De las Heras BB, Cortes Ramon y Cajal J, Galve Calvo E, et al. The economic burden of metastatic breast cancer in Spain. Eur J Hosp Pharm. 2020; 27(1):19-24.

46. Brandão M, Morais S, Lopes-Conceição L, Fontes F, Araújo N, Dias T, et al. Healthcare use and costs in early breast cancer: a patient-level data analysis according to stage and breast cancer subtype. ESMO Open. 2020;5(6):e000984.

47. Mery B, Rowinski E, Vallard A, et al. Advocacy for a new oncology research paradigm: the model of bevacizumab in triple-negative breast cancer in a French Cohort Study. Oncology. 2019;97(1):1-6.

48. Roman E, Cardoen B, Decloedt J, Roodhooft F. Variability in hospital treatment costs: a time-driven activity-based costing approach for early-stage invasive breast cancer patients. BMJ Open. 2020;10(7):e035389.

49. Skinner KE, Haiderali A, Huang M, Schwartzberg LS. Assessing direct costs of treating metastatic triple-negative breast cancer in the USA. J Comp Effect Res. 2020;10(2):109-18.

50. Parikh RC, Niyazov A, Esterberg E, et al. Real-world patient demographics, treatment patterns and healthcare resource utilization (HRU) among Human Epidermal Growth Factor Receptor 2 Negative (HER2-) Advanced Breast Cancer (ABC) patients with BRCA1/2 Mutations (BRCA1/2MUT). Value Health. 2020;23:S79

51. Valachis A, Nyström P, Fredriksson I, Wennstig AK, Ahlgren J. Treatment patterns, risk for hospitalization and mortality in older patients with triple negative breast cancer. J Geriatr Oncol. $2021 ; 12(2): 212-8$

52. Tabah A, Copher R, Huggar D, Tian M, Mougalian SS. Abstract P2-08-09: Treatment patterns and costs of metastatic triple negative breast cancer (mTNBC) in US women: a retrospective cohort study of first-line chemotherapy. Cancer Res. 2020;80(4 Suppl):P2-08-9.

53. Sieluk J, Haiderali A, Huang M, et al. PCN248 productivity costs associated with disease recurrence among surgically-treated patients with triple-negative breast cancer. Value in Health. 2021;24:S66.

54. Adams S, Diéras V, Barrios CH, et al. Patient-reported outcomes from the phase III IMpassion130 trial of atezolizumab plus nabpaclitaxel in metastatic triple-negative breast cancer. Ann Oncol. 2020;31(5):582-9.

55. Huang M, Haiderali A, Hu P, Mejia J. PCN308 health utility in patients with previously treated metastatic TNBC. Value in Health. 2020;23:S78.

56. Rugo H, Quek R, Ettl J, et al. 2920 Patient-reported outcomes (PRO) in patients (pts) with advanced breast cancer and a germline BRCA1/2 mutation (gBRCAm) receiving talazoparib (TALA) vs physician's choice chemotherapy treatment (PCT): A focus on the EMBRACA triple negative (TNBC) subpopulation. Ann Oncol. 2018;29(8):VIII91.

57. Schmid P, Haiderali A, Mejia J, et al. 141P Impact of pembrolizumab versus chemotherapy on health-related quality of life in patients with metastatic triple negative breast cancer. Ann Oncol. 2020;31:S65-6.

58. Swisher AK, Abraham J, Bonner D, Gilleland D, Hobbs G, Kurian $\mathrm{S}$, et al. Exercise and dietary advice intervention for survivors of triple-negative breast cancer: effects on body fat, physical function, quality of life, and adipokine profile. Support Care Cancer. 2015;23(10):2995-3003.

59. Anders C, Deal AM, Abramson V, Liu MC, Storniolo AM, Carpenter JT, et al. TBCRC 018: Phase II study of iniparib in combination with irinotecan to treat progressive triple negative breast cancer brain metastases. Breast Cancer Res Treat. 2014;146(3):557-66.

60. Anders CK, Deal AM, Abramson VG, Liu MC, Storniolo AM, Carpenter JT, et al. TBCRC 018: Phase II study of iniparib plus chemotherapy to treat triple-negative breast cancer (TNBC) central nervous system (CNS) metastases (mets). J Clin Oncol Conf. 2013;31(15):515.

61. Shen A, Qiang W, Wang Y, Chen Y. Quality of life among breast cancer survivors with triple negative breast cancer - role of hope, self-efficacy and social support. Eur J Oncol Nurs. 2020;46:1771.

62. Vadaparampil ST, Christie J, Donovan K, Kim J, Augusto B, Holt $\mathrm{C}$, et al. Health-related quality of life in black breast cancer patients with Triple Negative Breast Cancer (TNBC). In: Cancer Epidemiology Biomarkers and Prevention Conference: 9th AACR Conference on the Science of Cancer Health Disparities in Racial/ Ethnic Minorities and the Medically Underserved United States. 2017;26(2):B26.

63. Vadaparampil ST, Christie J, Donovan KA, Kim J, Augusto B, Kasting M, et al. Health-related quality of life in Black breast cancer survivors with and without triple-negative breast cancer (TNBC). Breast Cancer Res Treat. 2017;163(2):331-42.

64. Mocerino C, Letizia A, Taddeo M, Gambardella A. Tolerability of bevacizumab in elderly patients with "triple-negative" metastatic breast cancer. G Gerontol. 2012;60(3):149-53.

65. Aaronson NK, Ahmedzai S, Bergman B, et al. The European organization for research and treatment of cancer QLQ-C30: a quality-of-life instrument for use in international clinical trials in oncology. J Natl Cancer Inst. 1993;85(5):365-76.

66. Sprangers MA, Groenvold M, Arraras JI, et al. The European Organization for Research and Treatment of Cancer breast cancerspecific quality-of-life questionnaire module: first results from a three-country field study. J Clin Oncol. 1996;14(10):2756-68.

67. Cocks K, King MT, Velikova G, Fayers PM, Brown JM. Quality, interpretation and presentation of European Organisation for Research and Treatment of Cancer quality of life questionnaire core 30 data in randomised controlled trials. Eur J Cancer. 2008;44(13):1793-8.

68. Brady MJ, Cella DF, Mo F, et al. Reliability and validity of the functional assessment of cancer therapy-breast quality-of-life instrument. J Clin Oncol. 1997;15(3):974-86.

69. Eton DT, Cella D, Yost KJ, et al. A combination of distributionand anchor-based approaches determined minimally important differences (MIDs) for four endpoints in a breast cancer scale. J Clin Epidemiol. 2004;57(9):898-910.

70. Traina TA, Jones LW, Blinder V, et al. Abstract P5-12-09: Patientreported outcomes (PROs) during one year of adjuvant enzalutamide for the treatment of early stage androgen receptor positive (AR+) triple negative breast cancer. Cancer Res. 2020;80(4 Suppl):P5-12-09.

71. Naidoo S FM, Paly VF, Hansen R, Sidhu MK, Smith I. Targeted literature review of advanced/metastatic triple-negative breast cancer burden of illness. Value Health. 2017;20(5):360.

72. Fu A, Breznen B, Fazeli MS, Hux M, Kumar N, Davis C. PCN58 humanistic and economic burdens of triple-negative breast cancer: a literature review. Value Health. 2019;22:S67.

73. Frederickson A, Haiderali A, Zhang J, Chang A, Huang M. PCN468 systematic review of utility estimates in triple-negative breast cancer patients. Value Health. 2019;22:S527-8.

74. Frederickson A, Haiderali A, Zhang J, Chang A, Huang M. PCN132 systematic review of economic burden in triple-negative breast cancer patients. Value Health. 2019;22:S461. 


\section{Authors and Affiliations}

Min Huang ${ }^{1}$ (D) Amin Haiderali ${ }^{1} \cdot$ Grace E. Fox $^{2} \cdot$ Andrew Frederickson $^{2} \cdot$ Javier Cortes $^{3,4} \cdot$ Peter A. Fasching $^{5}$. Joyce O'Shaughnessy ${ }^{6}$

1 Merck \& Co., Inc., Kenilworth, NJ, USA

2 PRECISIONheor, New York, NY, USA

3 International Breast Cancer Center (IBCC), Barcelona, Spain

4 Vall d'Hebron Institute of Oncology (VHIO), Barcelona, Spain
5 Department of Gynecology and Obstetrics, Comprehensive Cancer Center Erlangen, EMN, University Hospital Erlangen, Friedrich-Alexander University Erlangen, Nuremberg, Erlangen, Germany

6 Baylor University Medical Center, Texas Oncology and US Oncology, Dallas, TX, USA 